The consequences of time-phased order releases on two $\mathrm{M} / \mathrm{M} / 1$ queues in series

Diederik Claerhout and Nico Vandaele

DEPARTMENT OF DECISION SCIENCES AND INFORMATION MANAGEMENT (KBI) 


\title{
The Consequences of Time-Phased Order Releases on two M/M/1 Queues in Series
}

\author{
Diederik Claerhout $^{\mathrm{ac}}$ and Nico Vandaele ${ }^{\mathrm{bc}}$ \\ ${ }^{a}$ Department of Business Studies, Lessius University College \\ diederik.claerhout@lessius.eu \\ ${ }^{\mathrm{b}}$ Faculty of Business and Economics, K.U.Leuven Campus Kortrijk \\ nico.vandaele@kuleuven-kortrijk.be \\ ${ }^{\mathrm{c}}$ Research Center for Operations Management, K.U.Leuven \\ Department of Decision Sciences and Information Management \\ diederik.claerhout@econ.kuleuven.be,nico.vandaele@econ.kuleuven.be
}

June 15, 2009

\begin{abstract}
A key characteristic of MRP applications includes the coordination of assembly and purchased component requirements by time-phased order releases. In the literature on order review and release strategies, timephased order releases are described as a worthy alternative to load limited release mechanisms. This paper initializes the development of a stochastic model that quantifies the consequences of time-phased order releases on the stochastic system behavior. This is done by introducing them in an open queueing network composed of two M/M/1 stations. The core of the analysis is focused on the modified flow variability which is specified by the second-order stationary departure process at the first station in the routing. It is a process characterized by a negligible autocorrelation. Based on the stationary-interval method and the asymptotic method, we propose an approximating renewal process for the modified departure process. The modelling efforts provide interesting conclusions and practical insights on some coordination issues in stochastic multi-echelon systems.
\end{abstract}

\section{Introduction}

Despite a variety of problems, the planning of manufacturing supply chains is still dominated by the utilization of models based on materials requirements planning (MRP) [19]. In these MRP applications, the coordination of assembly and purchased component requirements is simplified by setting time-phased order releases at each level of the bill of material [22]. To ensure that the multilevel 
dependent demand relationship is permanently linked, early release contradicts with the MRP release logic [16]. Orders will never be released until the release date is reached to avoid part shortages at the associated lower level bill of material when related parts at the higher level should be processed. In a more general way, a rigid release plan yields a higher degree of material traceability and a reduced system variability which simplifies the coordination of assembly operations, purchased component requirements, outsourced operations, operations requiring specific tools or specialized workforce... This is an important issue as international supply chains with global sourcing becomes more and more complex [23].

Controlling the workload on the shop floor, time-phased order releases are also proposed as a worthy alternative to load limited release mechanisms in the literature on order review and release strategies, [1] and [26]. Load limited release mechanisms or finite loading techniques are fine-tuning order releases by comparing the shop or machine loadings and the desired loadings. By contrast, time-phased order releases or infinite loading techniques are releasing orders at predetermined release dates regardless of the current loadings. While most research focuses on load limited release mechanisms, the greater simplicity of time-phased order releases, certainly in complex assembly systems, has made time-phased release mechanisms rather popular among industrial practitioners [1].

Another incentive to set time-phased order releases makes sense from a cost perspective, [27] and [7]. Assuming forbidden early departures, which were introduced in the literature by Kanet and Christy [14], order releases can be planned in order to minimize early completion holding costs and late completion tardy penalties. For example, if the cost rate per unit of time for carrying the order increases as the order moves from step to step, as is usually the case due to additional value being added, we may be better off setting an order release at each step, instead of planning only one release at the beginning of the routing [11]. Song et al. describe why these cost optimizing release plans should be executed very strictly [20]. First, early departures may be good in the local sense but may be not in the global sense. Secondly, rescheduling causes further deviation from the original plan. Thirdly, the interacting effects are very complicated and therefore difficult to estimate.

We conclude that there are several reasons to set and respect time-phased order releases in a supply chain. Unfortunately, time-phased order releases also have a major drawback. Quoted lead times will be inflated because a lower level of risk pooling is expected. A short cycle time on one workstation may result in an additional waiting time for a release authorization and may no longer compensate long cycle times in successive steps. The superiority of risk pooling was numerically illustrated by Vandaele and Lambrecht [21] when they compared an aggregate MRP setting with a traditional MRP setting. Instead of buffering each level of the bill of material separately against uncertainty and variability, their aggregate MRP concept buffers one aggregate level including different levels of the original bill of material. An evaluation of the advantages and disadvantages of time-phased order releases forces itself. 
What is the increase of the expected cycle time when time-phased order releases are set between two stations? Do infinite loading techniques always reduce the system variability? Will time-phased order releases ever increase the expected waiting time at the next station? Which factors do influence the impact of time-phased order releases on quoted lead times? Getting answers to those questions is not easy because a stochastic model of the behavior of manufacturing systems managed by time-phased order releases is missing. To the best of our knowledge there was only one attempt to develop queueing network models for systems controlled by time-phased order releases. In [4], Buzacott modelled the behavior of MRP controlled systems by using linked queueing network models developed for Kanban controlled systems. The equivalence between MRP controlled systems and Kanban controlled systems was realized by using specific blocking rules. Unfortunately, suitable solution techniques for these complex blocking mechanisms are never developed. Most papers studying time-phased order releases or infinite loading techniques use simulation experiments ([16], [8] and references in [26]). Other studies bypass the difficulty of quantifying the consequences of time-phased order releases on cycle time distrubutions by neglecting it ([27], [11] and [7]). One argues that initial release policies can be updated by using empirically observed cycle time distributions until convergence is obtained. A more refined approach to set release times combines the stochastic approximation method with the perturbation analysis technique ([12] and [20]).

In this paper, we analyze the consequences of time-phased order releases by introducing them in an open queueing network model. In Section 2, we introduce the concept of time-phased order releases by describing the queueing network under study and conclude that the modified departure process needs further research. The foundation for the characterization of this modified departure process is laid in Section 3 with the definition of a probability tree. The resulting renewal-interval distribution of the stationary-interval approximation and corresponding moments are computed in Sections 4 and 5. Because we only observe a second-order stationary departure process during the validation of the stationary-interval approximation in Section 6, the autocorrelation of the modified departure process is studied in Section 7 . In section 8, we propose two refined renewal approximations by letting the renewal-interval distribution of the departure process be a convex combination of the stationary-interval approximation and the asymptotic approximation. We finish the paper with some final conclusions and directions for further research in Section 9.

\section{Problem Description}

In Figure 1, a very basic environment is depicted. A make-to-order manufacturing system is composed of two single-machine workstations and produces only one type of product. The batch size equals one unit and there are no setup times. The processing times at both workstations are exponentially distributed with, respectively, $\mu_{1}$ and $\mu_{2}$ as parameters. Customer orders are characterized 
by an order quantity equal to one unit and a promised delivery date. Early deliveries are undesired and the required probability of on-time delivery equals 95 percent. To control the workload in the system, orders are only released when they become urgent. Order releases are based on lead time off-setting. Each order is released exactly LT12 time units before the promised delivery date. Here, LT12 represents a quoted lead time, the 95th percentile of the time to flow through the whole system. Assuming a Poisson process with parameter $\lambda$ for the promised delivery dates, the arrival process at the first workstation also equals a Poisson process with parameter $\lambda$.

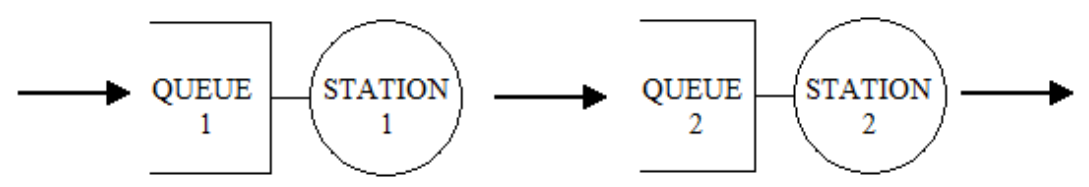

Figure 1: Basic M/M/1 - M/M/1 - line

In a stable system, when $\lambda<\mu_{1}$ and $\lambda<\mu_{2}$, the computation of LT12 is straightforward. Reminding Burke's theorem, stating that a Poisson arrival process driving an exponential server generates a Poisson departure process in a stable stationary queueing system [3], we recognize two successive $\mathrm{M} / \mathrm{M} / 1$ stations in Figure 1. Consequently, the probability density functions of the cycle times at both workstations are exponentially distributed: $f_{C T 1}($ ct 1$)=$ $\left(\mu_{1}-\lambda\right) e^{\left(\lambda-\mu_{1}\right) c t 1}$ and $f_{C T 2}(c t 2)=\left(\mu_{2}-\lambda\right) e^{\left(\lambda-\mu_{2}\right) c t 2}[15]$. The probability density function of the time to flow through the whole system is found by the following convolution:

$$
\begin{aligned}
f_{C T 12}(c t 12) & =\int_{0}^{c t 12} f_{C T 1}(c t 1) f_{C T 2}(c t 12-c t 1) d c t 1 \\
& =\frac{\left(e^{c t 12\left(\lambda-\mu_{2}\right)}-e^{c t 12\left(\lambda-\mu_{1}\right)}\right)\left(\lambda-\mu_{1}\right)\left(\lambda-\mu_{2}\right)}{\mu_{1}-\mu_{2}}
\end{aligned}
$$

Finally, LT12 is found by solving the following equation:

$$
\begin{gathered}
\int_{0}^{L T 12} f_{C T 12}(c t 12) d c t 12=0,95 \\
\Longleftrightarrow \frac{e^{L T 12\left(\lambda-\mu_{2}\right)}\left(\lambda-\mu_{1}\right)-e^{L T 12\left(\lambda-\mu_{1}\right)}\left(\lambda-\mu_{2}\right)}{\mu_{2}-\mu_{1}}=0,05
\end{gathered}
$$

When both workstations are characterized by the same utilization level, $\rho_{1}=\rho_{2}$ or $\lambda / \mu_{1}=\lambda / \mu_{2}$, previous computations become impossible due to a denominator equal to zero. The problem is solved by computing $f_{C T 12}(\operatorname{ct12})=$ 
$\int_{0}^{c t 12} f_{C T 1}(c t 1) f_{C T 1}(c t 12-c t 1) d c t 1=c t 12\left(\lambda-\mu_{1}\right)^{2} e^{\left(\lambda-\mu_{1}\right) c t 12} . \quad$ LT12 is found by solving $\left(1-L T 12\left(\lambda-\mu_{1}\right)\right) e^{L T 12\left(\lambda-\mu_{1}\right)}=0,05$.

In Figure 1, the transfer of orders between the workstations is free. When an order is finished on the first workstation, it will join the second queue without waiting for a release authorization. As discussed in Section 1, there are several motivations to manage the internal flow more rigorously. In Figure 2, the order transfer between the workstations is planned with a probability $P$ by setting intermediate release authorizations. When an order is finished early on the first workstation, it has to wait in the infinite "Wait for Release" buffer for its release authorization at the second station. These intermediate release authorizations are set by adding $L T 1$, the $P$ th percentile of the cycle time at the first station, to the release authorizations at the beginning of the routing. This quoted lead time $L T 1$ covers the expected cycle time at the first workstation and some allocated safety time to ensure an on-time delivery probability equal to $P$ percent to the second workstation. Consequently, the arrival time of an order at the second station is known with a probability equal to $P$ percent. It is important to note that the allocated safety time can only be used for delays at the first workstation. Any excess safety time is transformed in an additional waiting time in the "Wait for Release" buffer.

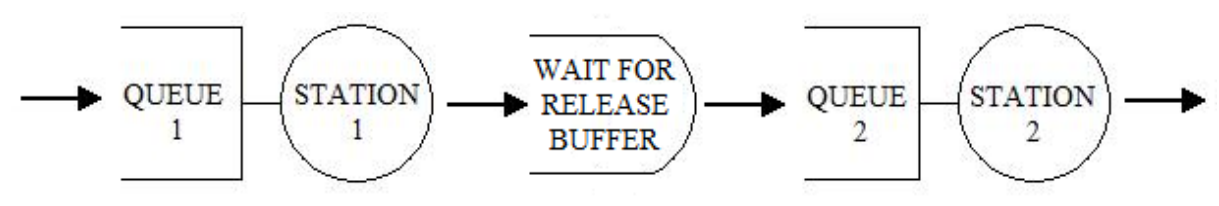

Figure 2: System with Intermediate Release Authorizations

On the right side of Figure 3 we observe how intermediate release authorizations modify the probability density function of the time interval between the initial release authorization and the arrival at the second queue. Values below $L T 1=\ln (1-P) /\left(\lambda-\mu_{1}\right)$ are no longer possible. More difficult to quantify is the departure process at the "Wait for Release" buffer, or equivalently the arrival process at the second workstation. The latter will be the focus of this paper and is important to quantify $L T 12$, the 95th percentile of the time to flow through the whole system, given an intermediate release authorization.

\section{Definition of Probability Tree}

The characterization of the departure process at the "wait for release" buffer in Figure 2 is founded on the definition of a probability tree. Assuming a stationary single-product single-machine workstation without batching and blocking, the 


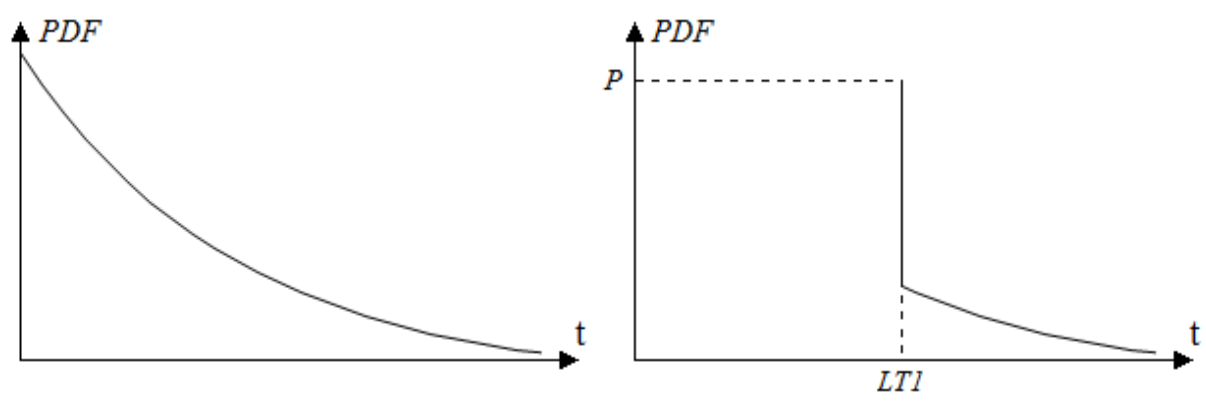

Figure 3: Modified Probability Density Function

following random variables are used:

$$
\begin{aligned}
A_{n} & =\text { time between } n^{\text {th }} \text { and }(n+1)^{t h} \text { arrival at "Queue 1" } \\
X_{n} & =\text { service time of } n^{t h} \text { customer at "Station 1" } \\
W_{n} & =\text { waiting time of } n^{\text {th }} \text { customer in "Queue 1" } \\
C T_{n} & =W_{n}+X_{n} \\
D_{n} & =\text { time between } n^{t h} \text { and }(n+1)^{t h} \text { departure at "Wait for release" }
\end{aligned}
$$

As depicted in Figure 3, LT1 is a constant quantifying the minimum time interval to flow through "Queue 1", "Station 1" and "Wait for release" buffer. We are now ready to observe $D_{n}$ when event type $C_{j 1}$ is occurring. Event type $C_{j 1}$ is defined by the following characteristics: the $n^{\text {th }}$ customer finds $j$ customers at the first workstation upon arrival; $C T_{n}<L T 1 ; A_{n}<C T_{n}$ and $X_{n+1}<A_{n}+L T-C T_{n}$. At the top of Figure 4, the $n^{\text {th }}$ customer arrives at the first station at epoch $a_{n}$ and has a cycle time $C T_{n}$. Because $C T_{n}<L T 1$, the $n^{\text {th }}$ customer has to wait for a release authorization and will only leave the "wait for release" buffer at epoch $d_{n}=a_{n}+L T 1$. The next customer arrives at epoch $a_{n+1}=a_{n}+A_{n}$.with $A_{n}<C T_{n}$, which results in positive waiting time $W_{n+1}$ at "Queue 1 ". Operations on the $(n+1)^{t h}$ customer can only start at epoch $a_{n}+C T_{n}=a_{n+1}+W_{n+1}$. In the last four lines it becomes clear that $X_{n+1}<A_{n}+L T 1-C T_{n}$ is equivalent with $W_{n+1}+X_{n+1}=C T_{n+1}<L T 1$ such that the $(n+1)^{t h}$ customer also has to wait for a release authorization. We observe that epoch $d_{n+1}=a_{n+1}+L T 1$. As a subsequence, $D_{n}=d_{n+1}-d_{n}=a_{n+1}+L T 1-a_{n}-L T 1=a_{n+1}-a_{n}=A_{n}$.

A similar analysis has to be made for an infinite number of other event types. Fortunately, nine types of events are returning for each $k$-value, the number of customers in the station upon arrival of the observed customer. In Figure 5 we define these nine types of events for $k=j$. At the top of this figure we recognize event type $C_{j 1}$ discussed in Figure 4 . For each of these event types, $D_{n}$ can be quantified in terms of $A_{n}, C T_{n}, X_{n+1}$ and $L T 1$. The results are reported in Table 1 . Before continuing our analysis, we simplify the probability 


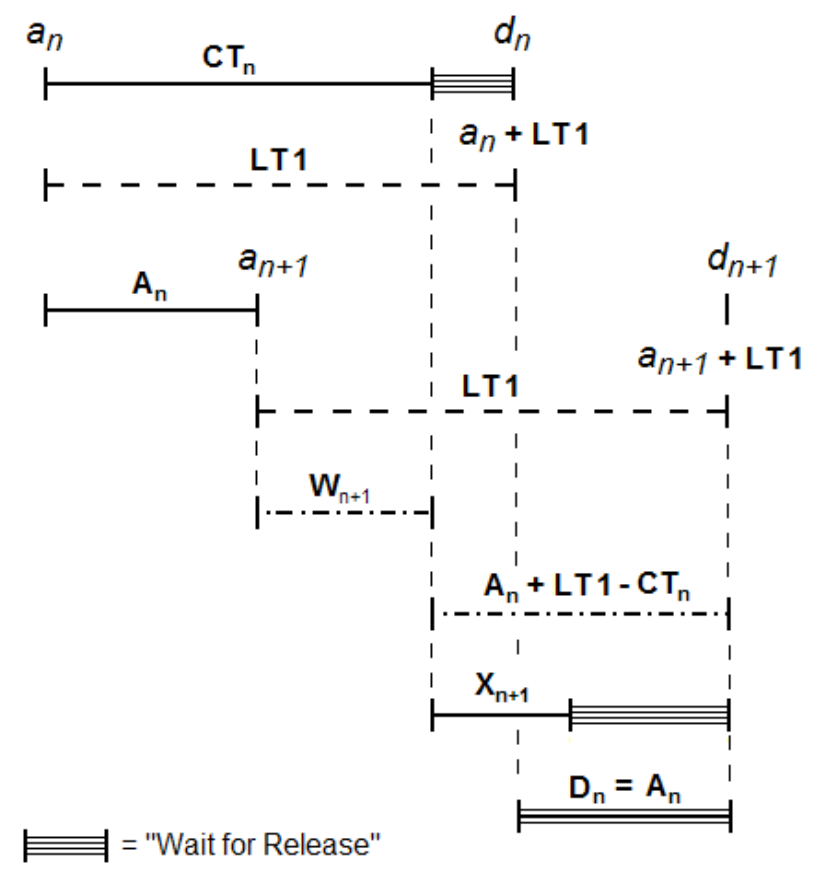

Figure 4: $D_{n}$ when event type $C_{j 1}$ is occuring

tree defined in Figure 5 to an equivalent tree depicted in Figure 6. In this simplified tree, we collect the event types $C_{k i}$ over all $k$-values in one event type $C_{i}$ and replace the conditional probability density function $f_{C T_{n}}\left(c t_{n} \mid k=j\right)$ by $f_{C T_{n}}\left(c t_{n}\right)=\sum_{j=0}^{\infty} P[k=j] * f_{C T_{n}}\left(c t_{n} \mid k=j\right)$ where $P[k=j]$ is the probability that $k=j$.

\section{Probability Density Function $f_{D_{n}}\left(d_{n}\right)$}

Assuming that all processes are renewal processes, the probability tree defined in Figure 6 allows the computation of $f_{D_{n}}\left(d_{n}\right)$, the renewal-interval distribution of the departure process at the "Wait for release" buffer. This function will be used to describe the modified arrival process at the second workstation. At the first workstation in Figure 2, a modified $L T 12$ value will not change the $\mathrm{M} / \mathrm{M} / 1$ characteristics of the station. The probability density functions $f_{A_{n}}\left(a_{n}\right), f_{X_{n+1}}\left(x_{n+1}\right)$ and $f_{C T_{n}}\left(c t_{n}\right)$ at the first workstation are known:

$$
\begin{aligned}
f_{A_{n}}\left(a_{n}\right) & =\lambda e^{-\lambda a_{n}} \\
f_{X_{n+1}}\left(x_{n+1}\right) & =\mu_{1} e^{-\mu_{1} x_{n+1}} \\
f_{C T_{n}}\left(c t_{n}\right) & =\left(\mu_{1}-\lambda\right) e^{\left(\lambda-\mu_{1}\right) c t_{n}}
\end{aligned}
$$




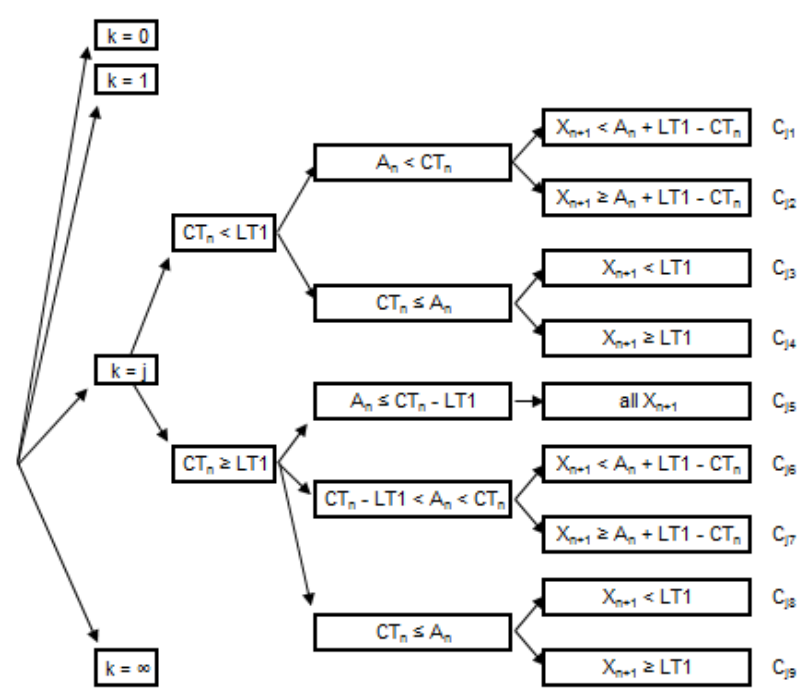

Figure 5: Release Authorization Probability Tree

We start by computing the probability of all types of events in Figure 6 .

$$
\begin{aligned}
P\left[C_{1}\right] & =\int_{0}^{L T 1} \int_{0}^{L t_{n} a_{n}+L T 1-c t_{n}} \int_{0} f_{X_{n+1}}\left(x_{n+1}\right) f_{A_{n}}\left(a_{n}\right) f_{C T_{n}}\left(c t_{n}\right) d x_{n+1} d a_{n} d c t_{n} \\
P\left[C_{2}\right] & =\int_{0}^{L T 1 c t_{n}} \int_{0}^{\infty} \int_{a_{n}+L T 1-c t_{n}}^{\infty} f_{X_{n+1}}\left(x_{n+1}\right) f_{A_{n}}\left(a_{n}\right) f_{C T_{n}}\left(c t_{n}\right) d x_{n+1} d a_{n} d c t_{n} \\
P\left[C_{3}\right] & =\int_{0}^{L T 1} \int_{c t_{n}}^{\infty} \int_{0}^{L T 1} f_{X_{n+1}}\left(x_{n+1}\right) f_{A_{n}}\left(a_{n}\right) f_{C T_{n}}\left(c t_{n}\right) d x_{n+1} d a_{n} d c t_{n} \\
P\left[C_{4}\right] & =\int_{0}^{L T 1} \int_{c t_{n}}^{\infty} \int_{L T 1}^{\infty} f_{X_{n+1}}\left(x_{n+1}\right) f_{A_{n}}\left(a_{n}\right) f_{C T_{n}}\left(c t_{n}\right) d x_{n+1} d a_{n} d c t_{n} \\
P\left[C_{5}\right] & =\int_{L T 1}^{\infty} \int_{0}^{c t_{n}-L T 1} f_{A_{n}}\left(a_{n}\right) f_{C T_{n}}\left(c t_{n}\right) d a_{n} d c t_{n} \\
P\left[C_{6}\right] & =\int_{L T 1 c t_{n}}^{\infty} \int_{L T 1}^{c t t_{n}} \int_{0}^{a_{n}+L T 1-c t_{n}} f_{X_{n+1}}\left(x_{n+1}\right) f_{A_{n}}\left(a_{n}\right) f_{C T_{n}}\left(c t_{n}\right) d x_{n+1} d a_{n} d c t_{n}
\end{aligned}
$$




\begin{tabular}{|l|l|}
\hline Event & Interdeparture Time \\
\hline$C_{j 1}$ & $D_{n}=A_{n}$ \\
\hline$C_{j 2}$ & $D_{n}=C T_{n}+X_{n+1}-L T 1$ \\
\hline$C_{j 3}$ & $D_{n}=A_{n}$ \\
\hline$C_{j 4}$ & $D_{n}=A_{n}+X_{n+1}-L T 1$ \\
\hline$C_{j 5}$ & $D_{n}=X_{n+1}$ \\
\hline$C_{j 6}$ & $D_{n}=A_{n}+L T 1-C T_{n}$ \\
\hline$C_{j 7}$ & $D_{n}=X_{n+1}$ \\
\hline$C_{j 8}$ & $D_{n}=A_{n}+L T 1-C T_{n}$ \\
\hline$C_{j 9}$ & $D_{n}=A_{n}+X_{n+1}-C T_{n}$ \\
\hline
\end{tabular}

Table 1: Interdeparture Time for all possible types of events

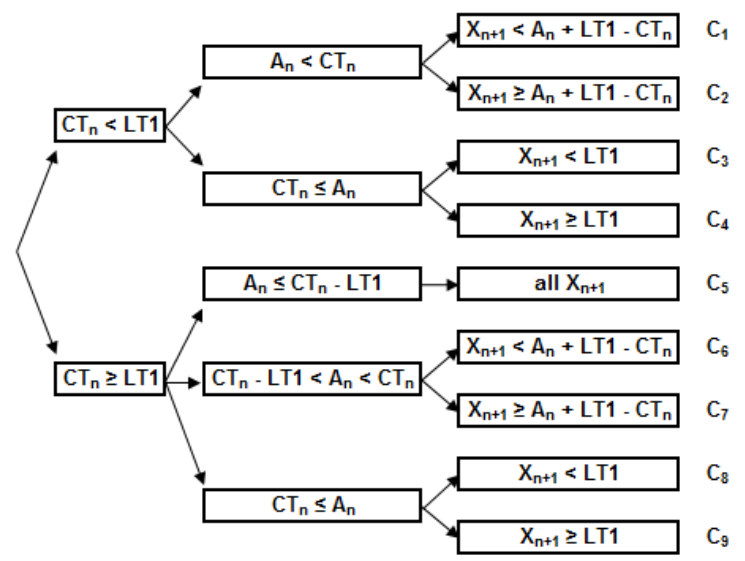

Figure 6: Simplified Probability Tree

$$
\begin{aligned}
P\left[C_{7}\right] & =\int_{L T 1 c t_{n}}^{\infty} \int_{L T 1 a_{n}+L T 1-c t_{n}}^{c t_{n}} \int_{X_{n+1}}^{\infty}\left(x_{n+1}\right) f_{A_{n}}\left(a_{n}\right) f_{C T_{n}}\left(c t_{n}\right) d x_{n+1} d a_{n} d c t_{n} \\
P\left[C_{8}\right] & =\int_{L T 1}^{\infty} \int_{c t_{n}}^{\infty} \int_{0}^{L T 1} f_{X_{n+1}}\left(x_{n+1}\right) f_{A_{n}}\left(a_{n}\right) f_{C T_{n}}\left(c t_{n}\right) d x_{n+1} d a_{n} d c t_{n} \\
P\left[C_{9}\right] & =\int_{L T 1 c t_{n}}^{\infty} \int_{L T 1}^{\infty} \int_{X_{X_{n+1}}}^{\infty}\left(x_{n+1}\right) f_{A_{n}}\left(a_{n}\right) f_{C T_{n}}\left(c t_{n}\right) d x_{n+1} d a_{n} d c t_{n}
\end{aligned}
$$

As required, summing all these probabilities yields a probability equal to one. Next, we determine $f_{D_{n}}\left(d_{n} \mid C_{i}\right)$, the conditional density function of $D_{n}$ assuming event type $C_{i}$ is observed.

$$
f_{D_{n}}\left(d_{n} \mid C_{1}\right)=
$$




$$
\begin{aligned}
& \begin{cases}\int_{d_{n}}^{L T 1 d_{n}+L T 1-c t_{n}} \int_{0}^{1-\frac{f_{X_{n+1}}\left(x_{n+1}\right) f_{A_{n}}\left(d_{n}\right) f_{C T_{n}}\left(c t_{n}\right)}{P\left[C_{1}\right]} d x_{n+1} d c t_{n}} & d_{n}<L T 1 \\
0 & d_{n} \geq L T 1\end{cases} \\
& f_{D_{n}}\left(d_{n} \mid C_{2}\right)=
\end{aligned}
$$

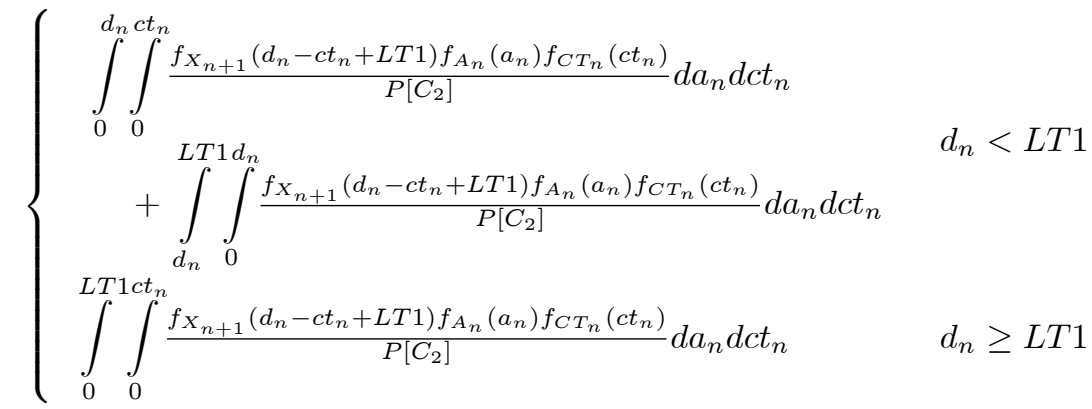

$$
\begin{aligned}
& f_{D_{n}}\left(d_{n} \mid C_{3}\right)= \\
& \begin{cases}\int_{0}^{d_{n}} \int_{0}^{L T 1} \frac{f_{X_{n+1}}\left(x_{n+1}\right) f_{A_{n}}\left(d_{n}\right) f_{C T_{n}}\left(c t_{n}\right)}{P\left[C_{3}\right]} d x_{n+1} d c t_{n} & d_{n}<L T 1 \\
\int_{0}^{L T 1} \int_{0}^{L T 1} \frac{f_{X_{n+1}}\left(x_{n+1}\right) f_{A_{n}}\left(d_{n}\right) f_{C T_{n}}\left(c t_{n}\right)}{P\left[C_{3}\right]} d x_{n+1} d c t_{n} & d_{n} \geq L T 1\end{cases} \\
& f_{D_{n}}\left(d_{n} \mid C_{4}\right)= \\
& \begin{cases}\int_{0}^{d_{n}} \int_{c t_{n}}^{d_{n}} \frac{f_{X_{n+1}}\left(d_{n}-a_{n}+L T 1\right) f_{A_{n}}\left(a_{n}\right) f_{C T_{n}}\left(c t_{n}\right)}{P\left[C_{4}\right]} d a_{n} d c t_{n} & d_{n}<L T 1 \\
\int_{0}^{L T 1} \int_{c t_{n}}^{d_{n}} \frac{f_{X_{n+1}}\left(d_{n}-a_{n}+L T 1\right) f_{A_{n}}\left(a_{n}\right) f_{C T_{n}}\left(c t_{n}\right)}{P\left[C_{4}\right]} d a_{n} d c t_{n} & d_{n} \geq L T 1\end{cases} \\
& f_{D_{n}}\left(d_{n} \mid C_{5}\right)= \\
& \begin{cases}\int_{L T 1}^{\infty} \int_{0}^{c t_{n}-L T 1} \frac{f_{X_{n+1}}\left(d_{n}\right) f_{A_{n}}\left(a_{n}\right) f_{C T_{n}}\left(c t_{n}\right)}{P\left[C_{5}\right]} d a_{n} d c t_{n} & d_{n}<L T 1 \\
\int_{L T 1}^{\infty} \int_{0}^{c t_{n}-L T 1} \frac{f_{X_{n+1}}\left(d_{n}\right) f_{A_{n}}\left(a_{n}\right) f_{C T_{n}}\left(c t_{n}\right)}{P\left[C_{5}\right]} d a_{n} d c t_{n} & d_{n} \geq L T 1\end{cases} \\
& f_{D_{n}}\left(d_{n} \mid C_{6}\right)= \\
& \begin{cases}\int_{L T 1}^{\infty} \int_{0}^{d_{n}} \frac{f_{X_{n+1}}\left(x_{n+1}\right) f_{A_{n}}\left(d_{n}-L T 1+c t_{n}\right) f_{C T_{n}}\left(c t_{n}\right)}{P\left[C_{6}\right]} d x_{n+1} d c t_{n} & d_{n}<L T 1 \\
0 & d_{n} \geq L T 1\end{cases}
\end{aligned}
$$




$$
\begin{aligned}
& f_{D_{n}}\left(d_{n} \mid C_{7}\right)= \\
& \begin{array}{l} 
\begin{cases}\int_{L T 1}^{\infty} \int_{c t_{n}-L T 1}^{d_{n}-L T 1+c t_{n}} \frac{f_{X_{n+1}}\left(d_{n}\right) f_{A_{n}}\left(a_{n}\right) f_{C T_{n}}\left(c t_{n}\right)}{P\left[C_{7}\right]} d a_{n} d c t_{n} & d_{n}<L T 1 \\
\int_{L T 1}^{\infty} \int_{c t_{n}-L T 1}^{c t_{n}} \frac{f_{X_{n+1}}\left(d_{n}\right) f_{A_{n}}\left(a_{n}\right) f_{C T_{n}}\left(c t_{n}\right)}{P\left[C_{7}\right]} d a_{n} d c t_{n} & d_{n} \geq L T 1\end{cases} \\
\begin{cases}f_{D_{n}}\left(d_{n} \mid C_{8}\right)= & d_{n}<L T 1 \\
\int_{L T 1}^{0} \int_{0}^{L T 1} \frac{f_{X_{n+1}}\left(x_{n+1}\right) f_{A_{n}}\left(d_{n}-L T 1+c t_{n}\right) f_{C T_{n}}\left(c t_{n}\right)}{P\left[C_{8}\right]} d x_{n+1} d c t_{n} & d_{n} \geq L T 1\end{cases}
\end{array} \\
& f_{D_{n}}\left(d_{n} \mid C_{9}\right)= \\
& \begin{cases}0 & d_{n}<L T 1 \\
\int_{L T 1}^{\infty} \int_{c t_{n}}^{c t_{n}+d_{n}-L T 1} \frac{f_{X_{n+1}}\left(d_{n}-a_{n}+c t_{n}\right) f_{A_{n}}\left(a_{n}\right) f_{C T_{n}}\left(c t_{n}\right)}{P\left[C_{9}\right]} d a_{n} d c t_{n} & d_{n} \geq L T 1\end{cases}
\end{aligned}
$$

Finally, $f_{D_{n}}\left(d_{n}\right)$ is found by working out $\sum_{i=1}^{9} P\left[C_{i}\right] f_{D_{n}}\left(d_{n} \mid C_{i}\right)$. We obtain a different expression for $d_{n}<L T 1$ and $d_{n} \geq L T 1$ :

$$
\begin{aligned}
f_{D_{n}<L T 1}\left(d_{n}\right)= & \frac{e^{\lambda L T 1+\mu_{1} d_{n}} \lambda^{3}\left(\lambda-\mu_{1}\right)+e^{\mu_{1} d_{n}} \lambda^{3} \mu_{1}+e^{\mu_{1}\left(d_{n}+L T 1\right)} \lambda^{2} \mu_{1}\left(\mu_{1}-\lambda\right)}{L} \\
& +\frac{e^{\lambda\left(d_{n}+L T 1\right)} \mu_{1}^{3}\left(\mu_{1}-\lambda\right)-e^{\lambda L T 1}\left(\lambda^{2}-\mu_{1}^{2}\right)^{2}}{L} \\
& +\frac{e^{\lambda d_{n}} \lambda \mu_{1}^{2}\left(\mu_{1}-\mu_{1}^{2} d_{n}+\lambda\left(\mu_{1} d_{n}-2\right)\right)}{L} \\
f_{D_{n} \geq L T 1}\left(d_{n}\right)= & \frac{e^{\lambda L T 1+\mu_{1} d_{n}} \lambda^{4}-e^{\mu_{1}\left(d_{n}+L T 1\right)} \lambda^{3} \mu_{1}+e^{\lambda d_{n}+\left(\lambda+\mu_{1}\right) L T 1}\left(\lambda-\mu_{1}\right) \mu_{1}^{3}}{M} \\
& +\frac{e^{\mu_{1} d_{n}+\left(\lambda+\mu_{1}\right) L T 1}\left(\mu_{1}-\lambda\right) \lambda^{3}-e^{\mu_{1}\left(d_{n}+2 L T 1\right)}\left(\mu_{1}-\lambda\right) \mu_{1} \lambda^{2}}{M} \\
& -\frac{e^{\lambda d_{n}+\mu_{1} L T 1}(1+\lambda L T 1)\left(\lambda-\mu_{1}\right) \mu_{1}^{3}}{M}
\end{aligned}
$$

with $L=\left(\lambda \mu_{1}\left(\mu_{1}-\lambda\right)\right) /\left(e^{-\mu_{1} L T 1-\left(\lambda+\mu_{1}\right) d_{n}}\right)$ and $M=\left(\lambda \mu_{1}\left(\lambda-\mu_{1}\right)\right) /\left(e^{-2 \mu_{1} L T 1-\left(\lambda+\mu_{1}\right) d_{n}}\right)$. Satisfying the requirements for a probability density function, it can be checked that $f_{D_{n}}\left(d_{n}\right)$ is a nonnegative continuous function with

$$
\int_{0}^{\infty} f_{D_{n}}\left(d_{n}\right) d d_{n}=\int_{0}^{L T 1} f_{D_{n}<L T 1}\left(d_{n}\right) d d_{n}+\int_{L T 1}^{\infty} f_{D_{n} \geq L T 1}\left(d_{n}\right) d d_{n}=1
$$

for $\lambda<\mu_{1}$ and $d_{n} \geq 0$. In Figure 7 , the probability density function $f_{D_{n}}\left(d_{n}\right)$ is depicted for a numerical example with $\lambda=0.6, \mu_{1}=1$ and $L T 1=4$. 


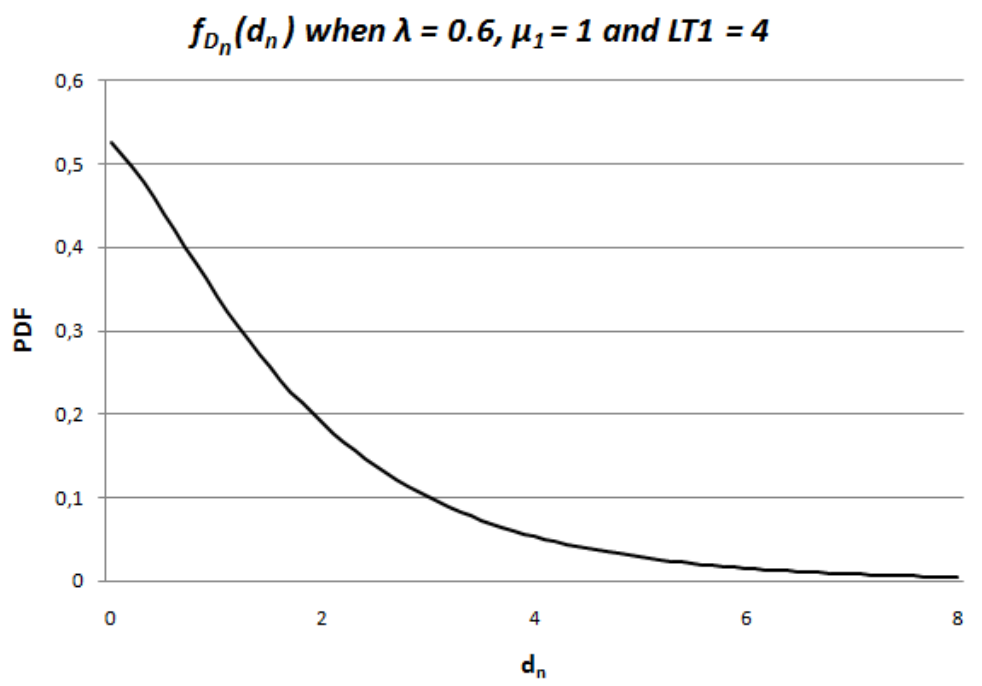

Figure 7: Probability Density Function $f_{D_{n}}\left(d_{n}\right)$

\section{Characteristics of Departure Process}

In queueing theory, special attention is paid to the first two moments of a random process. These can easily be computed for the departure process at the "Wait for release" buffer. For the first moment, we find that

$$
\int_{0}^{L T 1} d_{n} f_{D_{n}<L T 1}\left(d_{n}\right) d d_{n}+\int_{L T 1}^{\infty} d_{n} f_{D_{n} \geq L T 1}\left(d_{n}\right) d d_{n}=\frac{1}{\lambda}
$$

The expected interdeparture time equals the inverse arrival rate $\lambda$. This finding obeys the "Conservation of Material" law [13], stating that the departure rate of a stable workstation equals the arrival rate over the long run. Subtracting the squared first moment from the second moment and dividing the result by the squared first moment yields the squared coefficient of variation of the interdeparture time

$$
\begin{aligned}
S C V\left[D_{n}\right]= & \frac{e^{-2 L T 1 \mu_{1}}\left(2\left(1-e^{L T 1 \mu_{1}}\right) \lambda^{3}+2\left(e^{L T 1\left(\lambda+\mu_{1}\right)}-1\right) \lambda^{2} \mu_{1}\right)}{\mu_{1}^{2}\left(\lambda+\mu_{1}\right)} \\
& +\frac{e^{-2 L T 1 \mu_{1}}\left(e^{L T 1 \mu_{1}}\left(2-2 e^{L T 1 \lambda}+e^{L T 1 \mu_{1}}\right) \lambda \mu_{1}^{2}+e^{2 L T 1 \mu_{1}} \mu_{1}^{3}\right)}{\mu_{1}^{2}\left(\lambda+\mu_{1}\right)}
\end{aligned}
$$


Recalling form section 2 that $L T 1=\ln (1-P) /\left(\lambda-\mu_{1}\right)$, we can also express $S C V\left[D_{n}\right]$ in function of the probability $P$

$$
\begin{aligned}
S C V\left[D_{n}\right]= & \frac{2 \lambda^{2}\left(\lambda-\mu_{1}\right)(1-P)^{\frac{2 \mu_{1}}{\mu_{1}-\lambda}}+2 \lambda\left(\mu_{1}^{2}-\lambda^{2}\right)(1-P)^{\frac{\mu_{1}}{\mu_{1}-\lambda}}}{\mu_{1}^{2}\left(\lambda+\mu_{1}\right)} \\
& +\frac{\mu_{1}\left((2-2 P) \lambda^{2}+(2 P-1) \lambda \mu_{1}+\mu_{1}^{2}\right)}{\mu_{1}^{2}\left(\lambda+\mu_{1}\right)}
\end{aligned}
$$

We continue this section with a discussion of Figure 8. It reproduces $S C V\left[D_{n}\right]$

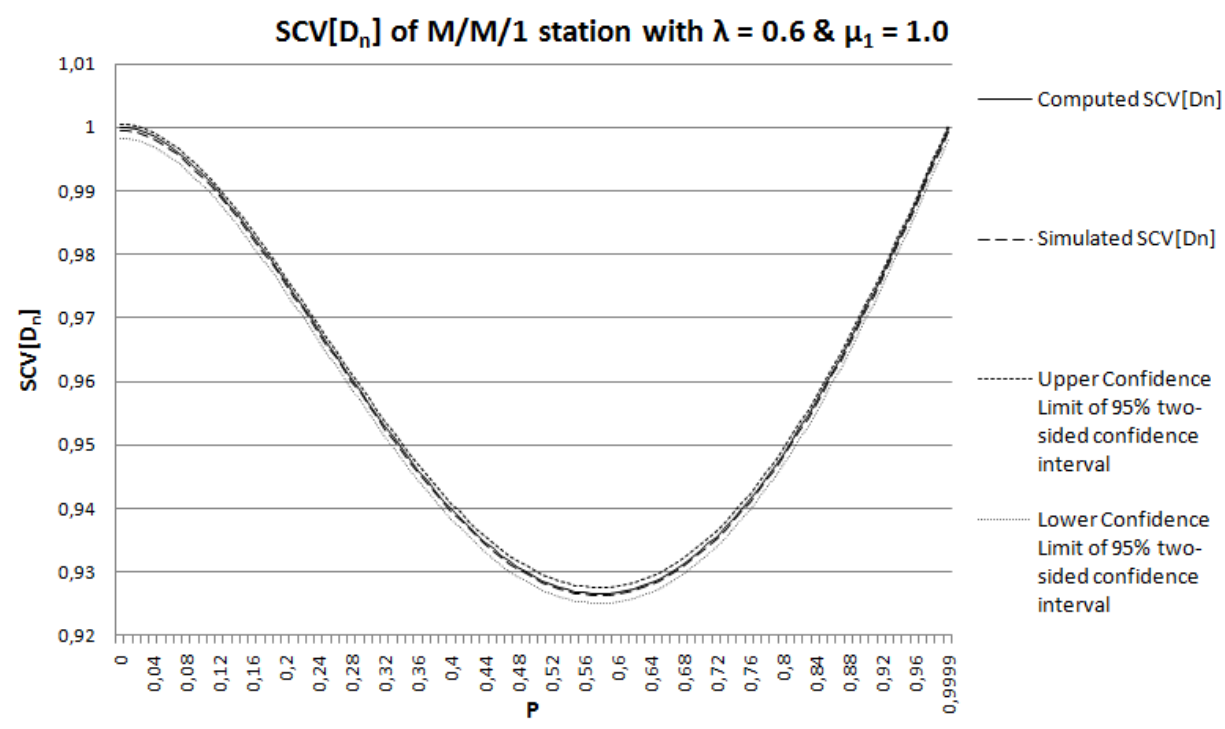

Figure 8: Modified $S C V\left[D_{n}\right]$ as a function of $P$

in function of $P$ when the first workstation in Figure 2 is characterized by an arrival rate $\lambda=0.6$ and a processing rate $\mu_{1}=1.0$. Although the analytical expression for $S C V\left[D_{n}\right]$ allows $0 \leq P \leq 1, P \in[0,0.9999]$ in order to complete Figure 8 with some simulation results. We start the interpretation of the results by discussing the extreme points:

- When $P=0$, then $L T 1=0$ and no intermediate release authorizations are set. We recognize the situation discussed in Figure 1. The $S C V\left[D_{n}\right]=1$ corresponds with the $S C V\left[D_{n}\right]$ of the Poisson departure process observed in Figure 1.

- When $P \rightarrow 1$, then $L T 1 \rightarrow \infty$ and all customers will have to wait for a release authorization. Each customer will leave the "Wait for release" buffer exactly $L T 1$ time units after the initial release. The $S C V\left[D_{n}\right] \approx 1$ approaches the $S C V\left[A_{n}\right]$ of the Poisson arrival process, which is equal to one. 
To explain the behavior in between these two extremes, we compute $S C V\left[D_{n}\right]$ as the weighted sum of the conditional $S C V\left[D_{n} \mid C_{i}\right]$ values where the $P\left[C_{i}\right]$ values are used as weights. Starting from the definition of the squared coefficient of variation, $S C V\left[D_{n}\right]$.

$$
\begin{aligned}
& =\frac{\left(\int_{-\infty}^{+\infty} d_{n}^{2} f_{D_{n}}\left(d_{n}\right) d d_{n}\right)-\left(\int_{-\infty}^{+\infty} d_{n} f_{D_{n}}\left(d_{n}\right) d d_{n}\right)^{2}}{\left(\int_{-\infty}^{+\infty} d_{n} f_{D_{n}}\left(d_{n}\right) d d_{n}\right)^{2}} \\
& =\frac{\sum_{i=1}^{9} P\left[C_{i}\right]\left(\int_{-\infty}^{+\infty} d_{n}^{2} f_{D_{n}}\left(d_{n} \mid C_{i}\right) d d_{n}-\left(\int_{-\infty}^{+\infty} d_{n} f_{D_{n}}\left(d_{n} \mid C_{i}\right) d d_{n}\right)^{2}\right)}{\left(\sum_{i=1}^{9} P\left[C_{i}\right] \int_{-\infty}^{+\infty} d_{n} f_{D_{n}}\left(d_{n} \mid C_{i}\right) d d_{n}\right)^{2}} \\
& +\frac{\sum_{i=1}^{9} P\left[C_{i}\right]\left(\int_{-\infty}^{+\infty} d_{n} f_{D_{n}}\left(d_{n} \mid C_{i}\right) d d_{n}\right)^{2}-\left(\sum_{i=1}^{9} P\left[C_{i}\right] \int_{-\infty}^{+\infty} d_{n} f_{D_{n}}\left(d_{n} \mid C_{i}\right) d d_{n}\right)^{2}}{\left(\sum_{i=1}^{9} P\left[C_{i}\right] \int_{-\infty}^{+\infty} d_{n} f_{D_{n}}\left(d_{n} \mid C_{i}\right) d d_{n}\right)^{2}} \\
& =\frac{\sum_{i=1}^{9} P\left[C_{i}\right]\left(\int_{-\infty}^{+\infty} d_{n}^{2} f_{D_{n}}\left(d_{n} \mid C_{i}\right) d d_{n}-\left(\int_{-\infty}^{+\infty} d_{n} f_{D_{n}}\left(d_{n} \mid C_{i}\right) d d_{n}\right)^{2}\right)}{\left(\sum_{i=1}^{9} P\left[C_{i}\right] \int_{-\infty}^{+\infty} d_{n} f_{D_{n}}\left(d_{n} \mid C_{i}\right) d d_{n}\right)^{2}} \\
& +\frac{\sum_{i=1}^{9} P\left[C_{i}\right]\left(\int_{-\infty}^{+\infty} d_{n} f_{D_{n}}\left(d_{n} \mid C_{i}\right) d d_{n}-\sum_{i=1}^{9} P\left[C_{i}\right] \int_{-\infty}^{+\infty} d_{n} f_{D_{n}}\left(d_{n} \mid C_{i}\right) d d_{n}\right)^{2}}{\left(\sum_{i=1}^{9} P\left[C_{i}\right] \int_{-\infty}^{+\infty} d_{n} f_{D_{n}}\left(d_{n} \mid C_{i}\right) d d_{n}\right)^{2}} \\
& =\sum_{i=1}^{9} P\left[C_{i}\right] S C V\left[D_{n} \mid C_{i}\right]
\end{aligned}
$$


with

$$
\begin{aligned}
& S C V\left[D_{n} \quad \mid \quad C_{i}\right]=\frac{\left(\int_{-\infty}^{+\infty} d_{n}^{2} f_{D_{n}}\left(d_{n} \mid C_{i}\right) d d_{n}-\left(\int_{-\infty}^{+\infty} d_{n} f_{D_{n}}\left(d_{n} \mid C_{i}\right) d d_{n}\right)^{2}\right)}{\left(\sum_{i=1}^{9} P\left[C_{i}\right] \int_{-\infty}^{+\infty} d_{n} f_{D_{n}}\left(d_{n} \mid C_{i}\right) d d_{n}\right)^{2}} \\
& +\frac{\left(\int_{-\infty}^{+\infty} d_{n} f_{D_{n}}\left(d_{n} \mid C_{i}\right) d d_{n}-\sum_{i=1}^{9} P\left[C_{i}\right] \int_{-\infty}^{+\infty} d_{n} f_{D_{n}}\left(d_{n} \mid C_{i}\right) d d_{n}\right)^{2}}{\left(\sum_{i=1}^{9} P\left[C_{i}\right] \int_{-\infty}^{+\infty} d_{n} f_{D_{n}}\left(d_{n} \mid C_{i}\right) d d_{n}\right)^{2}}
\end{aligned}
$$

In Figure 9, we find the decomposed $S C V\left[D_{n}\right]=\sum_{i=1}^{9} P\left[C_{i}\right] S C V\left[D_{n} \mid C_{i}\right]$ in function of $P$ for our $\mathrm{M} / \mathrm{M} / 1$ queue with arrival rate $\lambda=0.6$ and processing rate $\mu_{1}=1.0$. We observe that $S C V\left[D_{n}\right]$ initially decreases because the sum of the weighted variabilities of events $C_{9}$ and $C_{5}$ is decreasing more steeply than the increase of the sum of the other values. At higher $P$ values, $S C V\left[D_{n}\right]$ increases because the increasing weighted variabilities of events $C_{3}$ and $C_{1}$ are dominating. EvoWeightutIf we now study the corresponding $S C V\left[D_{n} \mid C_{i}\right]$ values in Figure 10, we notice that the decreasing impact of events $C_{9}$ and $C_{5}$ in Figure 9 can not be explained by decreasing variability levels. While $S C V\left[D_{n} \mid C_{5}\right]$ is constant, event $C_{9}$ is characterized by a very high and increasing $S C V\left[D_{n} \mid C_{9}\right]$. Similarly, the increasing impact of event $C_{1}$ in Figure 9 does not accord with its decreasing $S C V\left[D_{n} \mid C_{1}\right]$ in Figure 10. The answer has to be sought in Figure 11 which depicts the weights $P\left[C_{i}\right]$ in function of $P$. Clearly, at $P=0$, the departure process is dominated by events $C_{5}$ and $C_{9}$. At higher $P$ values, these positions are taken by events $C_{1}$ and $C_{3}$. Reminding the definitions of the events in Figure 6 , we observe that event $C_{9}$ is losing weight in favor of event $C_{3}$ with a detour to events $C_{8}$ and $C_{4}$ where $P\left[C_{4}\right]=P\left[C_{8}\right]$. Similarly, event $C_{5}$ is losing weight in favor of event $C_{1}$. In order to explain the behavior of $S C V\left[D_{n}\right]$, this latter shift is of less importance because Figure 10 shows us low and converging $S C V\left[D_{n} \mid\right.$ $\left.C_{5}\right]$ and $S C V\left[D_{n} \mid C_{1}\right]$ curves. We conclude that the decreasing $S C V\left[D_{n}\right]$ in Figure 8 is caused by the fact that the highly variable departure process of event $C_{9}$ is losing weight in favor of the more moderate departure processes of events $C_{3}$ and $C_{8}$. Unfortunately, at higher $P$ values, where event $C_{3}$ is dominating, $S C V\left[D_{n} \mid C_{3}\right]$ increases to the initial $S C V\left[D_{n} \mid C_{9}\right]$ value which explains the increasing $S C V\left[D_{n}\right]$ in Figure 8. Consequently, the behavior of $S C V\left[D_{n}\right]$ in Figure 8 is mainly caused by events characterized by idle time periods and small processing times. The flow variability reduces by imposing extra waiting times in the "Wait for release" buffer to customers without waiting times in "Queue $1 "$ and small processing times at "Station 1". More generally, the variability of 


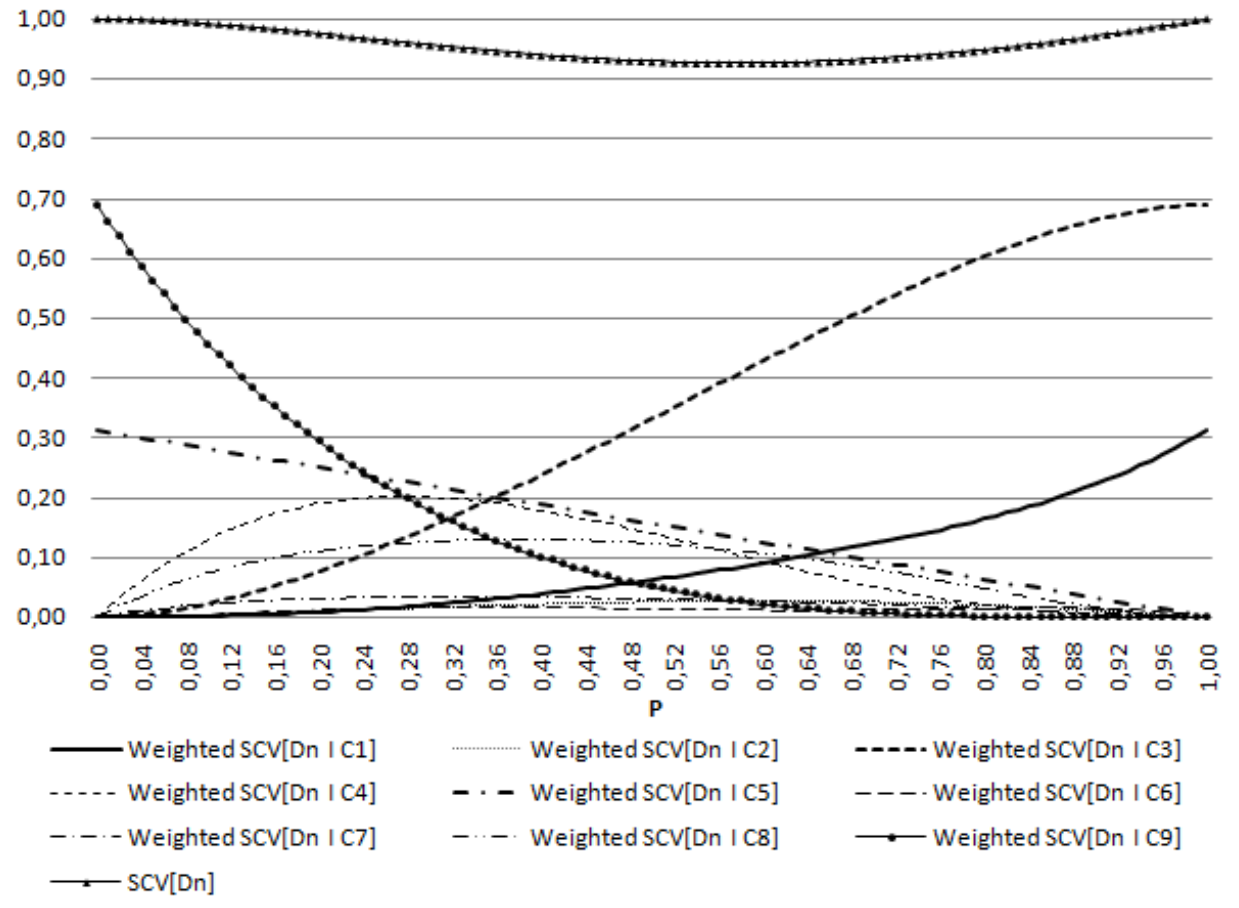

Figure 9: Weighted $S C V\left[D_{n} \mid C_{i}\right]$ Values as a function of $P$

the departure process reduces because short processing times are compensated by extra waiting times in the "Wait for release" buffer. However, at higher $P$ values, more and more customers have to wait for a release authorization. The departure process at the "Wait for release" buffer approaches the arrival process at "Queue 1". In Figure 8, the lowest variability $S C V\left[D_{n}\right]=0.9265$ is found at $P=0.5785$.

In the previous discussion, events $C_{3}, C_{4}, C_{8}$ and $C_{9}$ were playing an important role in the variability of the departure process. Because these events are characterized by idle time periods, a study of the impact of the utilization $\rho_{1}=\lambda / \mu_{1}$ on $S C V\left[D_{n}\right]$ forces itself. In Figure 12, we find the decomposed $S C V\left[D_{n}\right]=\sum_{i=1}^{9} P\left[C_{i}\right] S C V\left[D_{n} \mid C_{i}\right]$ in function of $\rho_{1}$ for an $\mathrm{M} / \mathrm{M} / 1$ queue with processing rate $\mu_{1}=1.0$ and $P=0.40$. We observe that $S C V\left[D_{n}\right]$ initially decreases because the sum of the weighted variabilities of events $C_{9}, C_{8}$ and $C_{4}$ is decreasing more steeply than the increase of the sum of the other values. At higher $P$ values, $S C V\left[D_{n}\right]$ increases because the increasing weighted variabilities of events $C_{5}$ and $C_{1}$ are dominating. If we now study the corresponding $S C V\left[D_{n} \mid C_{i}\right]$ values in Figure 13, we notice that the decreasing impact of events $C_{9}, C_{8}$ and $C_{4}$ in Figure 12 can not be explained by decreasing variability levels. The occurrence of idle time periods at these events strongly contributes 


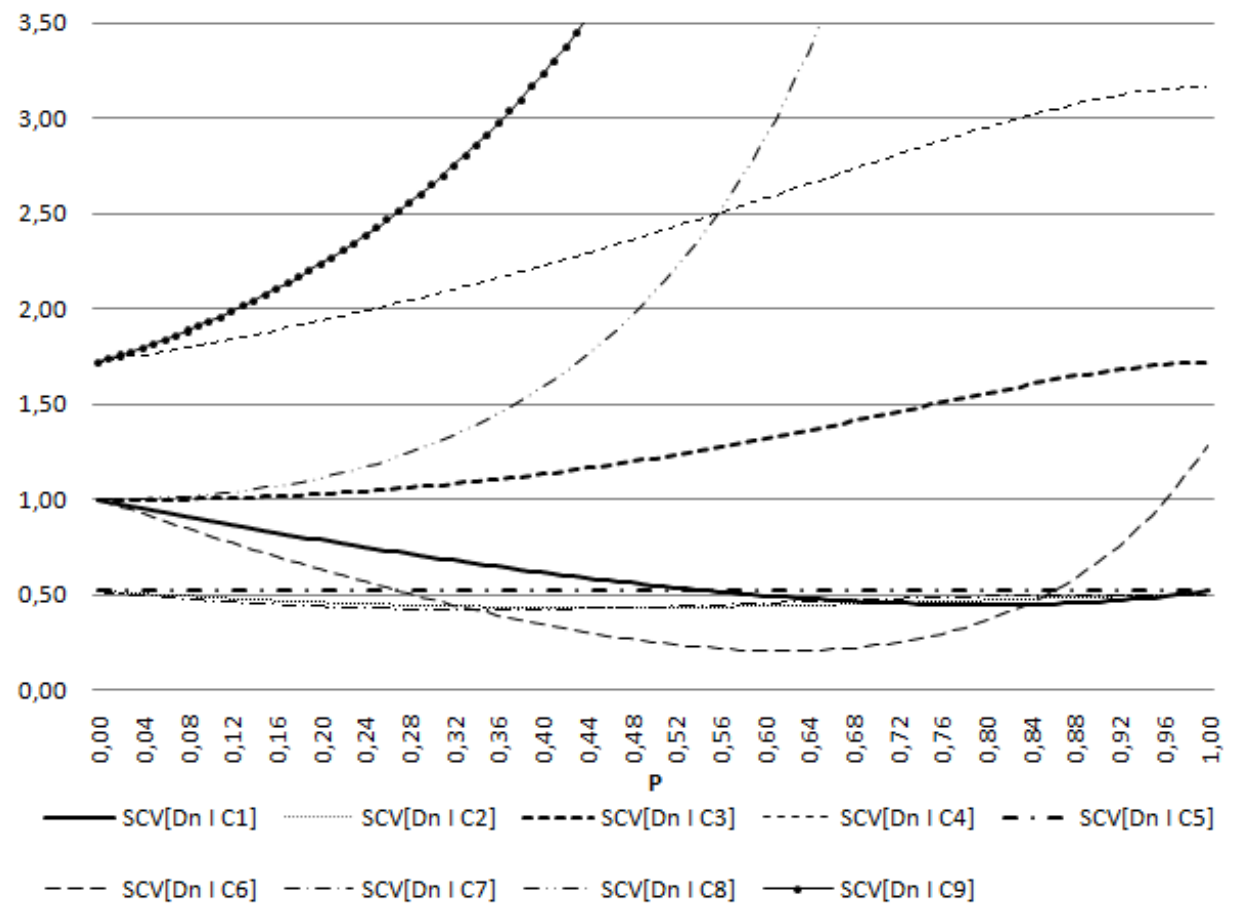

Figure 10: $S C V\left[D_{n} \mid C_{i}\right]$ as a function of $P$

to the variability of the departure process, an effect that becomes even more important at higher utilization levels. When the utilization approaches to zero, all $S C V\left[D_{n} \mid C_{i}\right]$ values converge to one. The departure processes of events $C_{1}, C_{3}, C_{4}, C_{6}, C_{8}$ and $C_{9}$ evolve to the Poisson arrival process while these of events $C_{2}, C_{5}$ and $C_{7}$ evolve to the Poisson service process. An analysis of the weights $P\left[C_{i}\right]$ in function of $\rho_{1}$ should clarify Figure 12, see Figure 14 where $P\left[C_{2}\right]=P\left[C_{6}\right]$ and $P\left[C_{4}\right]=P\left[C_{8}\right]$. Clearly, when $\rho_{1}$ increases, events $C_{9}, C_{8}$ and $C_{4}$ are losing weight because idle time periods are more seldom. Although event $C_{3}$ is also characterized by idle time periods, its weight initially increases because a higher utilization results in a higher $L T 1$ value, and as a consequence more extra waiting times in the "Wait for release" buffer. We conclude that the decreasing $S C V\left[D_{n}\right]$ in Figure 12 is caused by the fact that the highly variable departure processes of events $C_{9}, C_{8}$ and $C_{4}$ are losing weight in favor of events with more moderate departure processes. The underlying reason is the reduction of idle time periods. At higher utilization levels, the departure process is dominated by events $C_{1}$ and $C_{5}$ because the interarrival times become small compared to the long waiting times in "Queue 1". Because $S C V\left[D_{n} \mid C_{1}\right]$ and $S C V\left[D_{n} \mid C_{5}\right]$ evolve to one, $S C V\left[D_{n}\right]$ increases in Figure 12. More specific, the departure processes of events $C_{1}$ and $C_{5}$ evolve, respectively, to the Poisson 


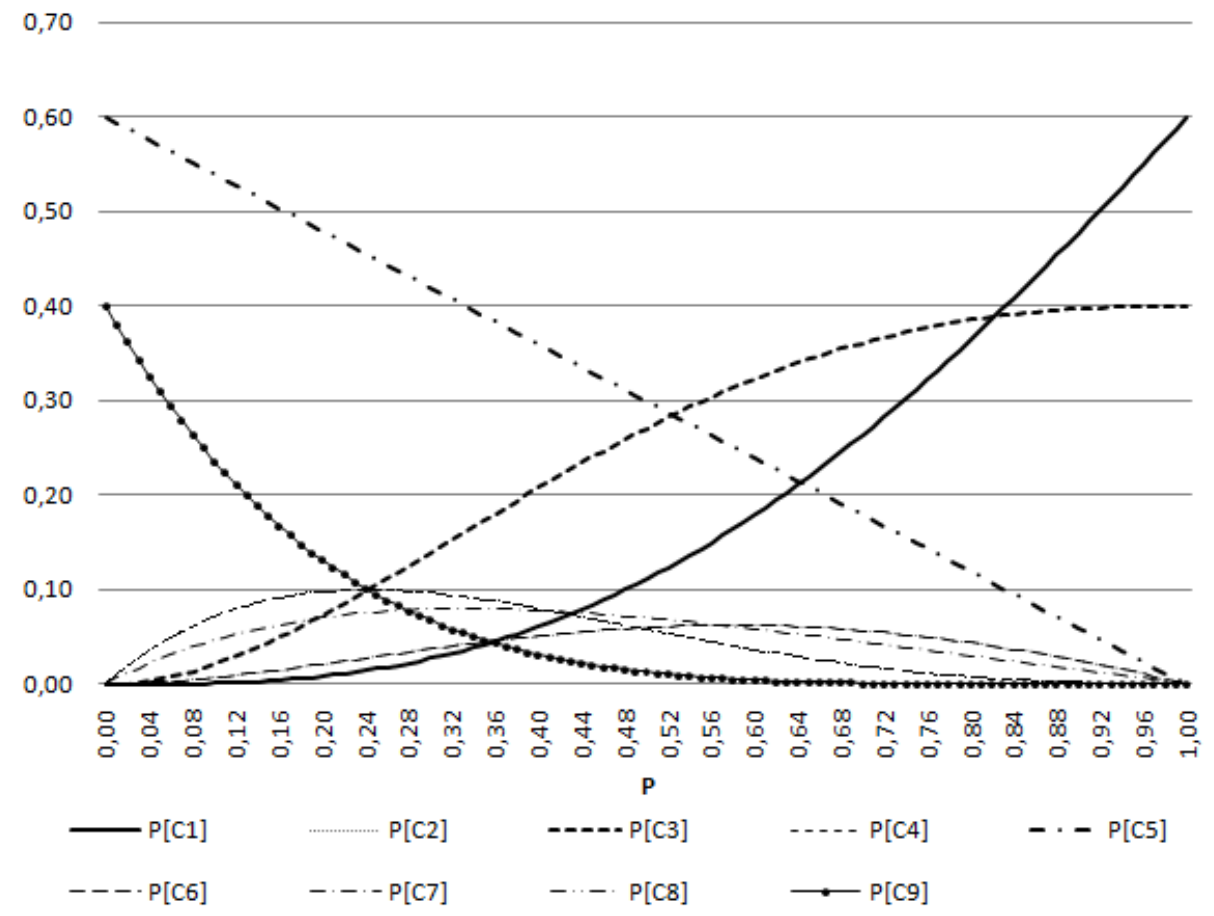

Figure 11: $P\left[C_{i}\right]$ as a function of $P$

arrival process at "Queue 1" and Poisson service process at "Station 1".

In Figure 15, depicting $S C V\left[D_{n}\right]$ in function $P$ and $\rho_{1}$, we join both dimensions. As illustrated above, the variability of the departure process initially reduces when short processing times are compensated by extra waiting times in the "Wait for release" buffer, $P \neq 0$. A downward trend that fades away when idle time periods dominate the departure process at low utilization levels or when long waiting times in "Queue 1" are dominating the system behavior at high utilization levels. Further, when $P$ approaches 1 , the departure process evolves to the Poisson arrival process at "Queue 1" because more and more customers have to wait for a release authorization. At heavy traffic stations, the minimum $S C V\left[D_{n}\right]$ occurs at a lower $P$ value because idle time periods, considerably distorting the heavy traffic departure process, are less important. The absolute minimum value of $S C V\left[D_{n}\right]$ equals 0.91499 and occurs at an utilization $\rho_{1}=0.7467$ and a $P$-value equal to 0.4674 . 


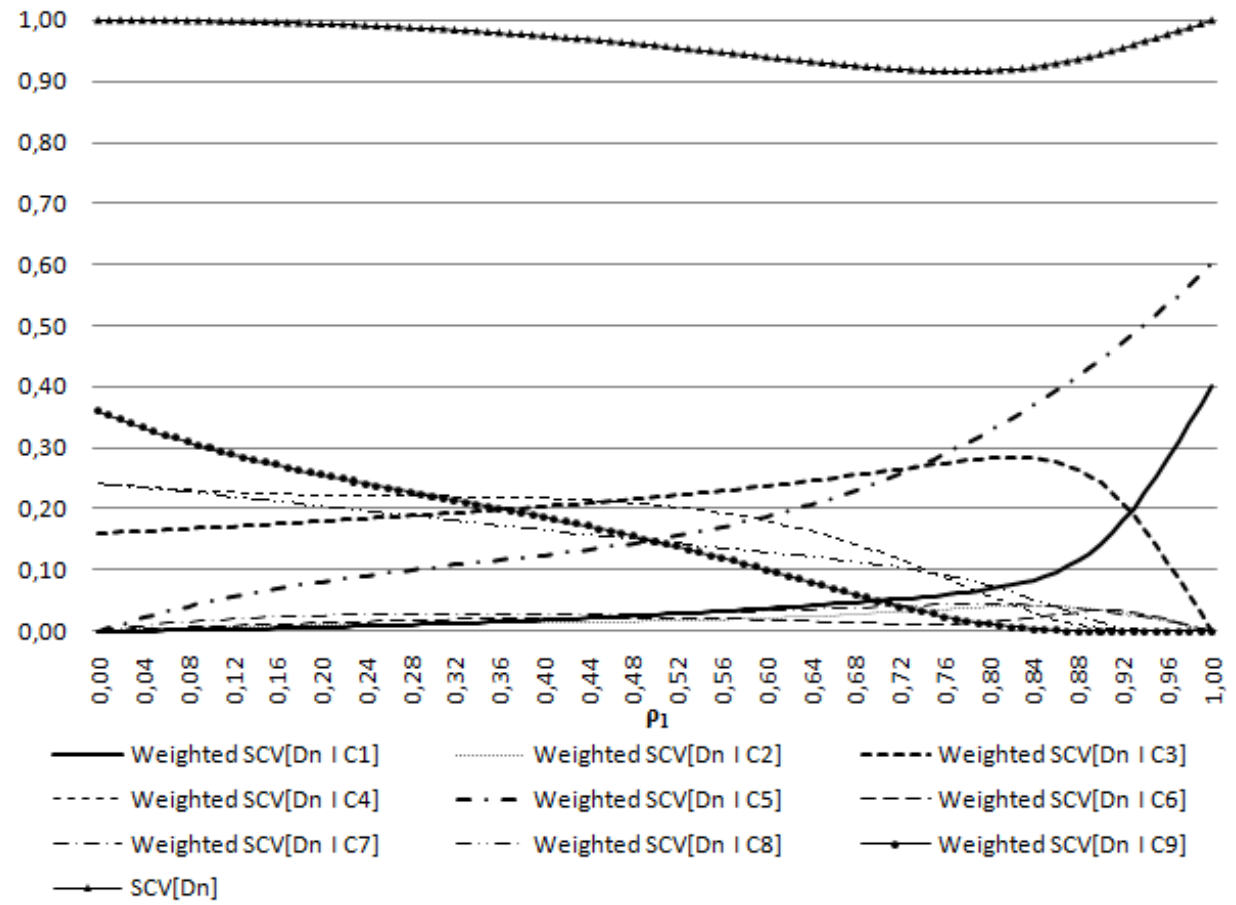

Figure 12: Weighted $S C V\left[D_{n} \mid C_{i}\right]$ Values as a function of $\rho_{1}$

\section{Impact of Intermediate Release Authorizations}

In this section, we finish the computation of $L T 12$, the 95 th percentile of the time to flow through the whole system in Figure 2. The arrival process at the second workstation is modelled by a renewal process with renewal-interval distribution $f_{D_{n}}\left(d_{n}\right)$, the probability density function of the interdeparture time at the "Wait for release" buffer discussed in section 4. Defining $D^{*}(s)$ to be the Laplace transform of $f_{D_{n}}\left(d_{n}\right)$ and searching for the unique root of $\sigma=$ $D^{*}\left(\mu_{2}-\mu_{2} \sigma\right)$ in the range $0<\sigma<1$, the probability density function of the cycle time at the second workstation $f_{C T 2}(c t 2)$ is found by the G/M/1 computation described in [15]. Unfortunately, we do not find a closed-form solution for the unique root of $\sigma=D^{*}\left(\mu_{2}-\mu_{2} \sigma\right)$ in the range $0<\sigma<1$. Our numerical example with arrival rate $\lambda=0.6$, processing rate $\mu_{1}=1.0$, processing rate $\mu_{2}=0.8$ and $L T 1=4$ or $P=0.7981$ yields an exponential distribution with parameter 0.205269 for $f_{C T 2}(c t 2)$. As explained in Figure 3 , the time to flow through the whole system may contain an extra waiting time in the "Wait for release" buffer. The probability density function of the cycle time at the first workstation, including the extra waiting time for a release 


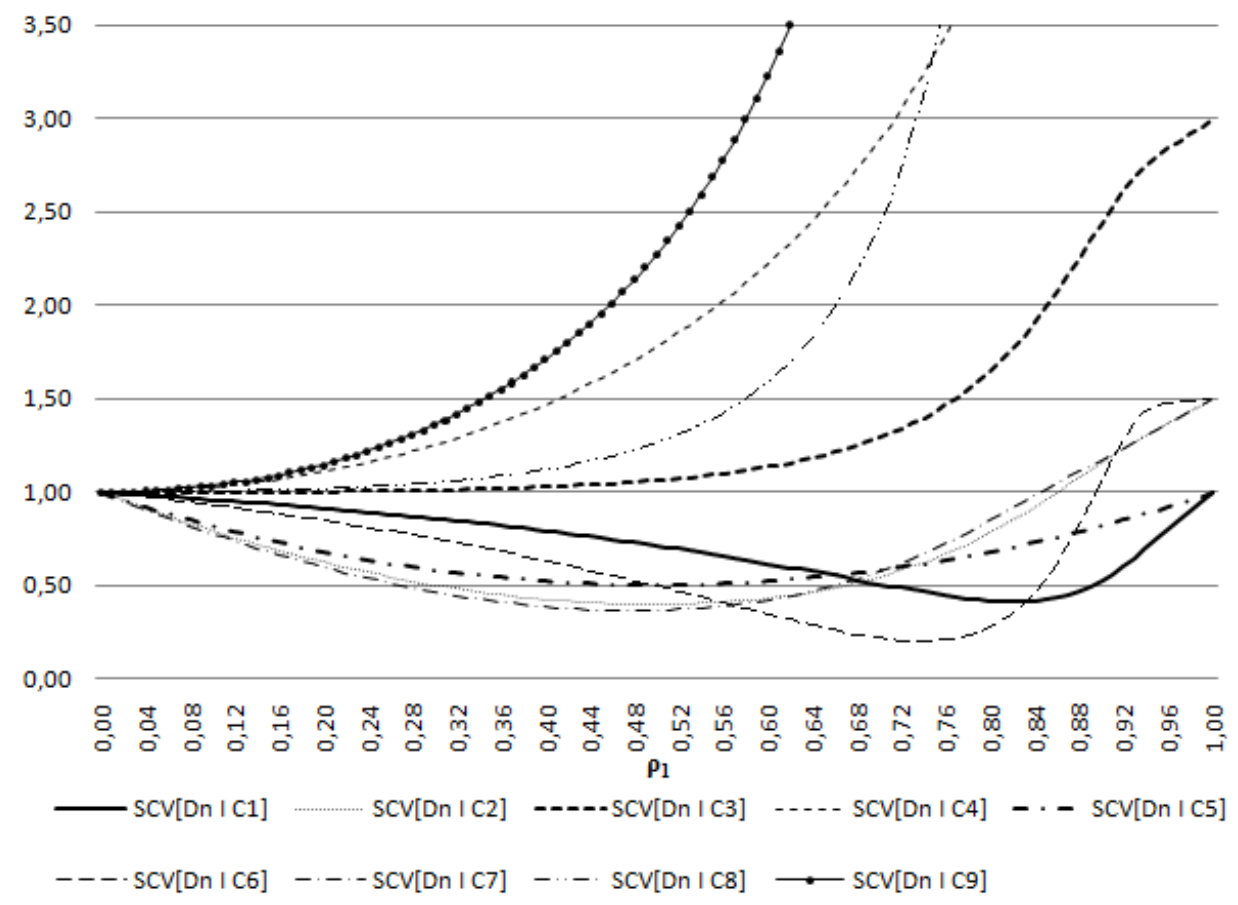

Figure 13: $S C V\left[D_{n} \mid C_{i}\right]$ as a function of $\rho_{1}$

authorization becomes

$$
f_{C T_{1}}\left(c t_{1}\right)=\left\{\begin{array}{lll}
0 & c t_{1}<L T 1 \\
& \left(\mu_{1}-\lambda\right) e^{\left(\lambda-\mu_{1}\right) c t 1}+P \delta\left(c t_{1}-L T 1\right) & c t_{1} \geq L T 1
\end{array}\right.
$$

with the Dirac delta distribution $\delta(t)$ defined as:

$$
\delta(t)= \begin{cases}\int_{-\infty}^{\infty} \delta(\tau) d \tau=1 & \text { when } t=0 \\ 0 & \text { when } t \neq 0\end{cases}
$$




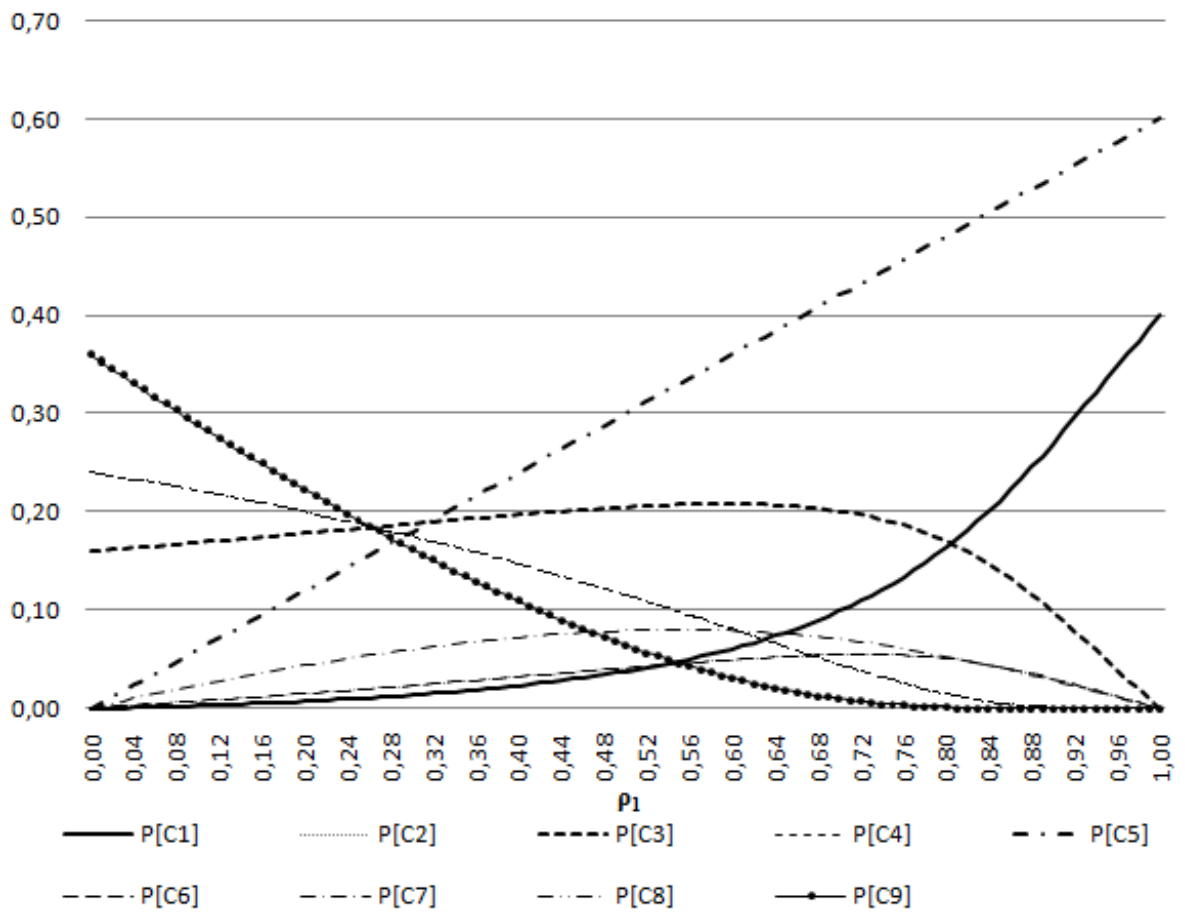

Figure 14: $P\left[C_{i}\right]$ as a function of $\rho_{1}$

The probability density function of the time to flow through the whole system is computed by the following convolution:

$$
\begin{aligned}
f_{C T_{12}}\left(c t_{12}\right)= & \int_{-\infty}^{+\infty} f_{C T_{1}}\left(c t_{12}-c t_{2}\right) f_{C T_{2}}\left(c t_{2}\right) d c t_{2} \\
= & \int_{0}^{c t_{12}-L T 1}\left(\mu_{1}-\lambda\right) e^{\left(\lambda-\mu_{1}\right)\left(c t_{12}-c t_{2}\right)} f_{C T_{2}}\left(c t_{2}\right) d c t_{2} \\
& +P \int_{0}^{c t_{12}-L T 1} \delta\left(c t_{12}-c t_{2}-L T 1\right) f_{C T_{2}}\left(c t_{2}\right) d c t_{2}
\end{aligned}
$$

Using the "Sifting Property" of the Dirac delta distribution, stating that $\int_{-\infty}^{+\infty} \delta(t-$ $\left.t_{0}\right) u(t) d t=u\left(t_{0}\right)$ which is proved in Appendix A, we find that $f_{C T_{12}}\left(c t_{12}\right)$ equals

$$
\int_{0}^{c t_{12}-L T 1}\left(\mu_{1}-\lambda\right) e^{\left(\lambda-\mu_{1}\right)\left(c t_{12}-c t_{2}\right)} f_{C T_{2}}\left(c t_{2}\right) d c t_{2}+P f_{C T_{2}}\left(c t_{12}-L T 1\right)
$$




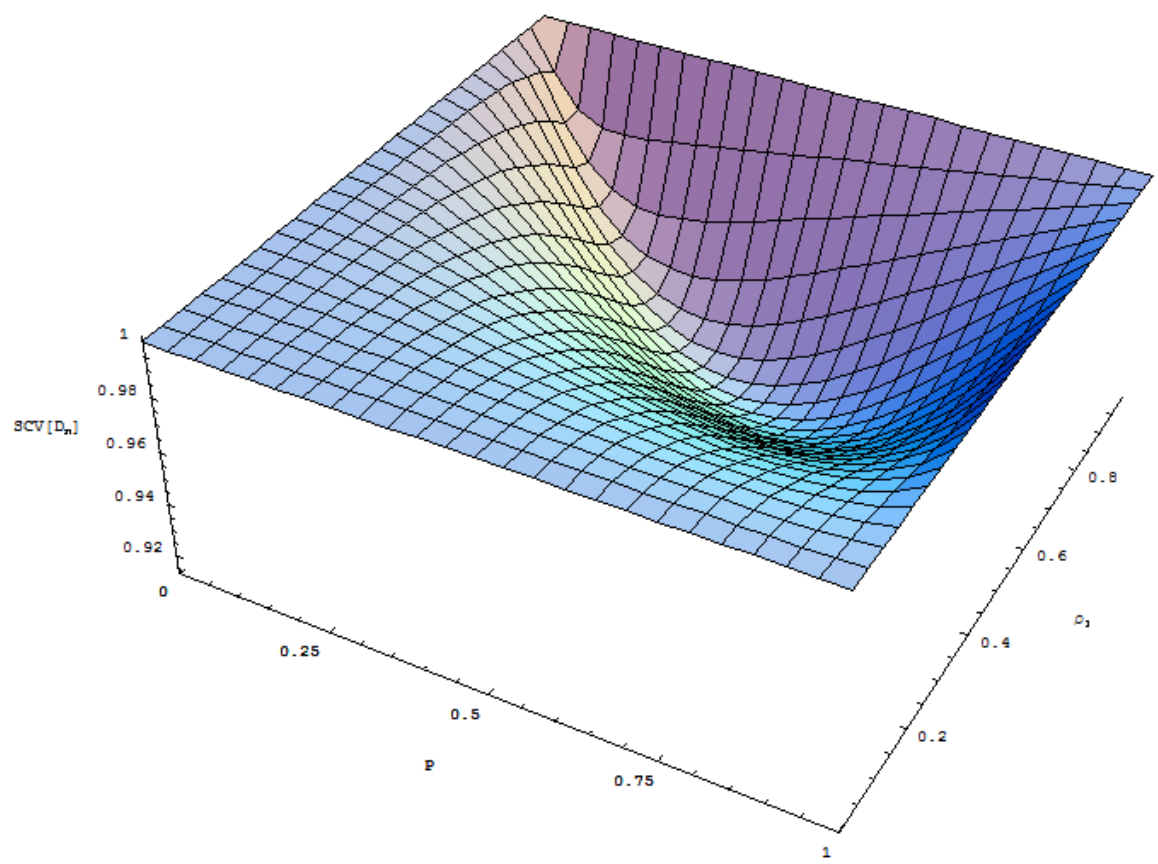

Figure 15: Modified $S C V\left[D_{n}\right]$ as a function of $P$ and $\rho_{1}$

for $c t_{12} \geq L T 1$ and zero for $c t_{12}<L T 1$. This $f_{C T_{12}}\left(c t_{12}\right)$ expression allows equal utilization levels at both workstations, $\rho_{1}=\rho_{2}$, as long as $0<P<1$. When $P=0$, we recall the solution described in section 2 . When $P=1$, the time to flow through the whole system becomes infinite. In Figure 16, two $f_{C T_{12}}\left(c t_{12}\right)$ curves are depicted for our numerical example with $\lambda=0.6, \mu_{1}=1.0$ and $\mu_{2}=0.8$ :

- The dashed curve $f_{C T_{12}}\left(c t_{12}\right)$ with $L T 1=0$ or $P=0.0000$. The situation without intermediate release authorizations as discussed in Figure 1.

- The full curve $f_{C T_{12}}\left(c t_{12}\right)$ with $L T 1=4$ or $P=0.7981$. The situation with intermediate release authorizations as discussed in Figure 2.

Clearly, the total cycle time increases when intermediate release authorizations are set. In Table 2, we summarize the expected time to flow through the whole system $E[C T 12]$ and the corresponding 95th percentile $L T 12$ for the manufacturing systems depicted in Figure 1 and 2 with $\lambda=0.6, \mu_{1}=1.0$ and $\mu_{2}=0.8$. In the first two rows, we compare the exact computation for $L T 1=0$ (Original) with the above described computations for $L T 1=4$ (Approx1). As observed in Figure 16, the planning of order transfers with a probability equal to $79.81 \%$, or equivalently setting $L T 1=4$, has its price. The expected total 


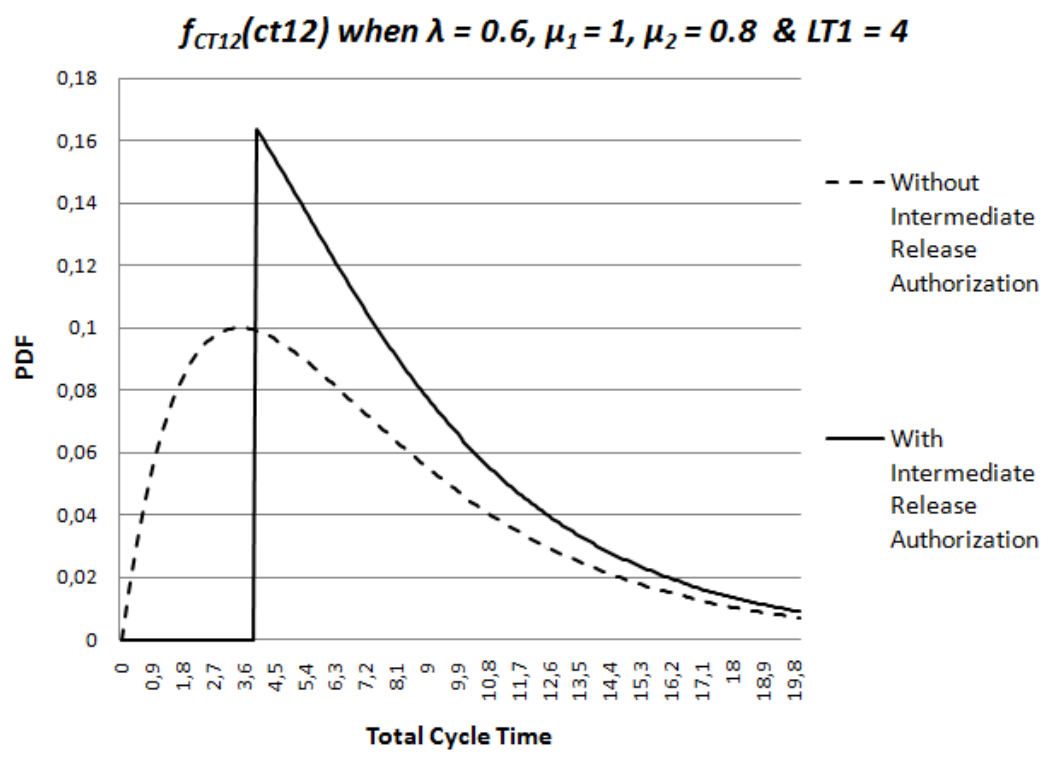

Figure 16: Effect of Intermediate Release Authorizations

cycle time $E[C T 12]$ and 95th percentile $L T 12$ increase, respectively, from 7.50 to 9.38 and from 18.38 to 19.49 time units. Falsely neglecting the modified arrival process at the second workstation and assuming an $\mathrm{M} / \mathrm{M} / 1$ station in Approx2, makes performance even worse. $E[C T 12]$ and $L T 12$ increase to 9.50 and 19.86. Unfortunately, when we compare the above described approximation (Approx1) with some simulation results (Lower Limit of two-sided 95\% Confdence Interval, Estimated Value Simulation and Upper Limit of two-sided 95\% Confidence Interval), we observe a significant deviation in Table 2. While the approximated value of $E[C T 12]=9.38$ coincides with the lower limit of the two-sided $95 \%$ confidence interval, the approximated value of $L T 12=19.49$ falls outside the confidence interval. The reason for this deviation has to be

\begin{tabular}{|c|c|c|}
\hline & $E[C T 12]$ & $L T 12$ \\
\hline Original & 7.50 & 18.38 \\
\hline Approx1 & 9.38 & 19.49 \\
\hline Approx2 & 9.50 & 19.86 \\
\hline LL 95\% C.I. & 9.38 & 19.69 \\
EVS & 9.40 & 19.74 \\
UL 95\% C.I. & 9.42 & 19.79 \\
\hline
\end{tabular}

Table 2: Impact of Intermediate Release Authorizations

sought in our approximation of the arrival process at the second workstation. 
We falsely assumed that the successive intervals in the departure process at the "Wait for release" buffer are independent. However, it seems that this departure process is only a second-order stationary process where the mean and variance are time-independent and the correlation depends on the time-distance between the pair of values but not on their position in time. In the next section, we study the autocorrelation in the interdeparture times at the "Wait for release" buffer.

\section{Autocorrelation of Departure Process}

In the past, several papers studied the autocorrelation of the departure process at a single server queue ([3], [5], [6], [25], [10], [9], [17] and references therein). In this section, we study the impact of the additional waiting times for a release authorization on the autocorrelation of the $\mathrm{M} / \mathrm{M} / 1$ departure process. Considering our second-order stationary, real-valued time series $\left\{D_{n}\right\}(n=0, \ldots, \infty)$ in discrete time, the pairs $\left(D_{n}, D_{n+\tau}\right)$ have the same distribution as $\left(D_{0}, D_{\tau}\right)$ and the autocorrelation function is defined as:

$$
\beta_{D_{n}}(\tau)=\frac{E\left[D_{n} D_{n+\tau}\right]-E\left[D_{n}\right]^{2}}{\operatorname{Var}\left[D_{n}\right]} \text { for } \tau=0,1, \ldots
$$

where $E\left[D_{n}\right]$ is the common mean and $\operatorname{Var}\left[D_{n}\right]$ the common variance of $D_{n}$ studied in section 5. Starting with the computation of the lag-1 correlation $\beta_{D_{n}}$ (1) requiring the joint moment of two successive intervals $E\left[D_{n} D_{n+1}\right]$, we study $D_{n+1}$ by extending the tree depicted in Figure 6 . For example, when event $C_{1}$ with $D_{n}=A_{n}$ is occurring in Figure 6, four relevant sub-events $Q_{i}$ can be distinguished for $D_{n+1}$, see Figure 17. Similarly, in Appendix B, the leaves of the other branches in Figure 6 are depicted. The corresponding quantification of $D_{n}$ and $D_{n+1}$, in terms of $A_{n}, A_{n+1}, C T_{n}, X_{n+1}, X_{n+2}$ and $L T 1$, for all subevents $Q_{i}$ can be found in Appendix C. This extended probability tree can now

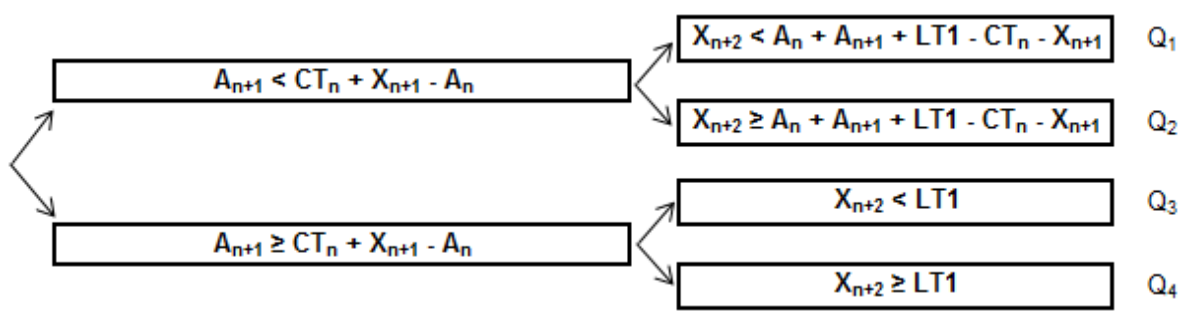

Figure 17: Sub-events of $C_{1}$ 
be used to compute the joint moment of two successive intervals $E\left[D_{n} D_{n+1}\right]$

$$
\begin{aligned}
& =\int_{-\infty}^{+\infty} \int_{-\infty}^{+\infty} d_{n} d_{n+1} f_{D_{n} D_{n+1}}\left(d_{n}, d_{n+1}\right) d d_{n} d d_{n+1} \\
& =\int_{-\infty}^{+\infty} \int_{-\infty}^{+\infty} d_{n} d_{n+1}\left[\sum_{i=1}^{41} P\left[Q_{i}\right] * f_{D_{n} D_{n+1}}\left(d_{n}, d_{n+1} \mid Q_{i}\right)\right] d d_{n} d d_{n+1} \\
& =\sum_{i=1}^{41} P\left[Q_{i}\right] \int_{-\infty}^{+\infty} \int_{-\infty}^{+\infty} d_{n} d_{n+1} f_{D_{n} D_{n+1}}\left(d_{n}, d_{n+1} \mid Q_{i}\right) d d_{n} d d_{n+1} \\
& =\sum_{i=1}^{41} P\left[Q_{i}\right] E\left[D_{n} D_{n+1} \mid Q_{i}\right]
\end{aligned}
$$

where the definitions in Figure 6 and Appendices B and C satisfy to compute $P\left[Q_{i}\right]$ and $E\left[D_{n} D_{n+1} \mid Q_{i}\right]$. For example, when the arrival and service process are both renewal processes with renewal-interval distributions $f_{A_{n+1}}(a)=f_{A_{n}}(a)$ and $f_{X_{n+1}}(x)=f_{X_{n+2}}(x)$, we obtain:

$$
\begin{aligned}
P\left[Q_{1}\right] & =\int_{0}^{L T} \int_{0}^{L c t_{n} U} \int_{0}^{U B_{1} U B_{2} U B_{0}} \int_{0}^{B_{3}} I_{P} d x_{n+2} d a_{n+1} d x_{n+1} d a_{n} d c t_{n} \\
E\left[D_{n} D_{n+1} \mid Q_{1}\right] & =\int_{0}^{L T 1 c t_{n} U B_{1} U B_{2} U B_{3}} \int_{0}^{B_{3}} \int_{0}^{2} I_{E} d x_{n+2} d a_{n+1} d x_{n+1} d a_{n} d c t_{n}
\end{aligned}
$$

with $I_{P}=f_{X_{n+1}}\left(x_{n+2}\right) f_{A_{n}}\left(a_{n+1}\right) f_{X_{n+1}}\left(x_{n+1}\right) f_{A_{n}}\left(a_{n}\right) f_{C T_{n}}\left(c t_{n}\right)$

$$
\begin{aligned}
I_{E} & =\left(a_{n} a_{n+1}\right) \frac{I_{P}}{P\left[Q_{1}\right]} \\
U B_{1} & =a_{n}+L T 1-c t_{n} \\
U B_{2} & =c t_{n}+x_{n+1}-a_{n} \\
U B_{3} & =a_{n}+a_{n+1}+L T 1-c t_{n}-x_{n+1}
\end{aligned}
$$

As discussed in section 4 , the probability density functions $f_{A_{n}}\left(a_{n}\right), f_{X_{n+1}}\left(x_{n+1}\right)$ and $f_{C T_{n}}\left(c t_{n}\right)$ at the first workstation in Figure 2 are known. Recalling form section 2 that $L T 1=\ln (1-P) /\left(\lambda-\mu_{1}\right)$, we find the following expression for the lag-1 correlation $\beta_{D_{n}}(1)$ in function of $P$ : 


$$
\begin{aligned}
& \frac{\lambda^{2}\left(-\lambda^{3}-\lambda^{2} \mu_{1}+3 \lambda \mu_{1}^{2}-\mu_{1}^{3}-2 \lambda \mu_{1}\left(\lambda+\mu_{1}\right) \ln (1-P)\right)}{N} \\
& +\frac{(1-P)^{\frac{\mu_{1}}{\lambda-\mu_{1}}} \lambda^{2}\left(\lambda-\mu_{1}\right)\left(-2(1-P)^{\frac{\lambda}{\lambda-\mu_{1}}} \mu_{1}^{2}+\left(\lambda+\mu_{1}\right)^{2}\right)}{N} \\
& +\frac{(1-P)^{\frac{\mu_{1}}{\lambda-\mu_{1}}} \lambda^{2} \mu_{1}\left(\lambda+\mu_{1}\right)^{2} \ln (1-P)}{N}
\end{aligned}
$$

with $N$ equal to:

$$
\begin{aligned}
& \left(\lambda+\mu_{1}\right)^{2}\left(-2 \lambda^{2}\left(\lambda-\mu_{1}\right)-(1-P)^{\frac{2 \mu_{1}}{\lambda-\mu_{1}}} \mu_{1}^{2}\left(\lambda+\mu_{1}\right)\right) \\
& +2(1-P)^{\frac{\mu_{1}}{\lambda-\mu_{1}}} \lambda\left(\lambda-\mu_{1}\right)\left(\lambda+\mu_{1}\right)^{2}\left(\lambda+\mu_{1}-(1-P)^{\frac{\lambda}{\lambda-\mu_{1}}} \mu_{1}\right)
\end{aligned}
$$

Contrary to the $S C V\left[D_{n}\right]$ expression in section 5 , this analytical expression for $\beta_{D_{n}}$ (1) does not allow a $P$-value equal to $100 \%$. Figure 18 reproduces $\beta_{D_{n}}(1)$ in function of $P \in[0,0.9999]$ for our numerical example with arrival rate $\lambda=0.6$ and processing rate $\mu_{1}=1.0$. Two findings have to be outlined:

- $P=0.6014$ yielding $\beta_{D_{n}}(1)=0$ does not correspond with $P=0.5785$ minimizing the variability $S C V\left[D_{n}\right]$.

- Two successive interdeparture intervals are almost uncorrelated: $-0.00648 \leq$ $\beta_{D_{n}}(1) \leq 0.00767$.

If we define the utilization $\rho_{1}=\lambda / \mu_{1}$ and set $\mu_{1}=1.0$, we can reproduce $\beta_{D_{n}}$ (1) for all $\left(P, \rho_{1}\right)$ settings, see Figure 19. Clearly, the impact of intermediate release authorizations on the lag- 1 correlation of the $\mathrm{M} / \mathrm{M} / 1$ departure process is negligible for any setting. While $\left(P=0.5213, \rho_{1}=0.8369\right)$ minimizes $\beta_{D_{n}}(1)$ to -0.0150 , the maximum of $\beta_{D_{n}}(1)$ equals 0.0081 and occurs at $\left(P=0.4284, \rho_{1}=\right.$ $0.5060)$. The question is whether $\beta_{D_{n}}(\tau)$ is also negligible for $\tau>1$. It is a difficult question to answer because a general expression for $\beta_{D_{n}}(\tau)$ can not be found. The latter is caused by the fact that the system loses its memoryless property. For example, a huge processing time in the past may void the impact of release authorizations on all subsequent customers. In other words, it is not possible to define an underlying markov chain for the stochastic departure process at the "Wait for release" buffer. Consequently, the modified departure process does not satisfy the characteristics of a Markov Renewal Process that generalizes many well-known random processes, including the Poisson process, Markov process and renewal process, and takes account of correlations. Each increase of $\tau$ requires additional nodes in the probability tree computing the joint moment $E\left[D_{n} D_{n+\tau}\right]$. That is why we study $\beta_{D_{n}}(2), \beta_{D_{n}}(3), \beta_{D_{n}}(4), \beta_{D_{n}}(5)$, $\beta_{D_{n}}(10), \beta_{D_{n}}(20)$ and $\beta_{D_{n}}$ (50) for different utilization levels $\rho_{1}$ using discreteevent simulations. The results in Figures 20-22 illustrate that $\max \left[\beta_{D_{n}}(1)\right] \geq$ $\max \left[\beta_{D_{n}}(\tau)\right]$ and $\min \left[\beta_{D_{n}}(1)\right] \leq \min \left[\beta_{D_{n}}(\tau)\right]$ for all $\tau$ and $\rho_{1}$ values. We 


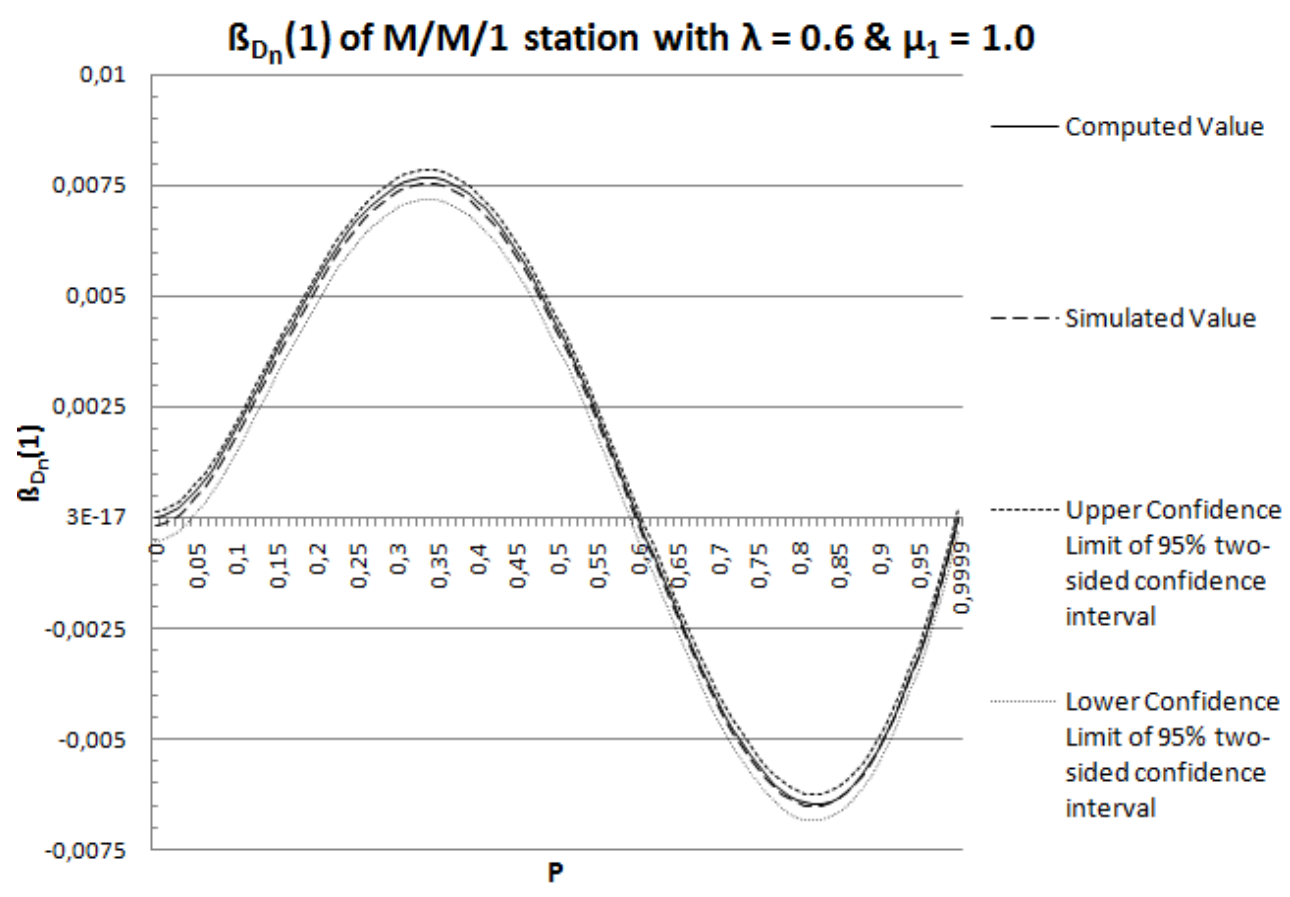

Figure 18: $\beta_{D_{n}}(1)$ as a function of $P$

conclude that the autocorrelation of the modified departure process is negligible. Important, this lack of correlation does not mean that successive intervals in the departure process at the "Wait for release" buffer are independent. One may only conclude that there is no linear dependence [18]. In the next section, we propose an alternative approximation for the modified departure process $D_{n}$ in order to better take into account the dependence among successive intervals.

\section{Refined Approximation of Departure Process}

In [24], Whitt described two basic methods for approximating a general stochastic process by a renewal process: the stationary-interval method and the asymptotic method. With the stationary-interval method, the renewal-interval distribution is chosen to match the distribution of the stationary interval between points in the process being approximated. Because this method does not take account of dependence among successive intervals, he also discussed the asymptotic method which matches the long-run behavior of the process being approximated. The essential idea behind this asymptotic method is that renewal processes can be used as approximating processes without ignoring the dependence among the intervals in the process being approximated. Refined 


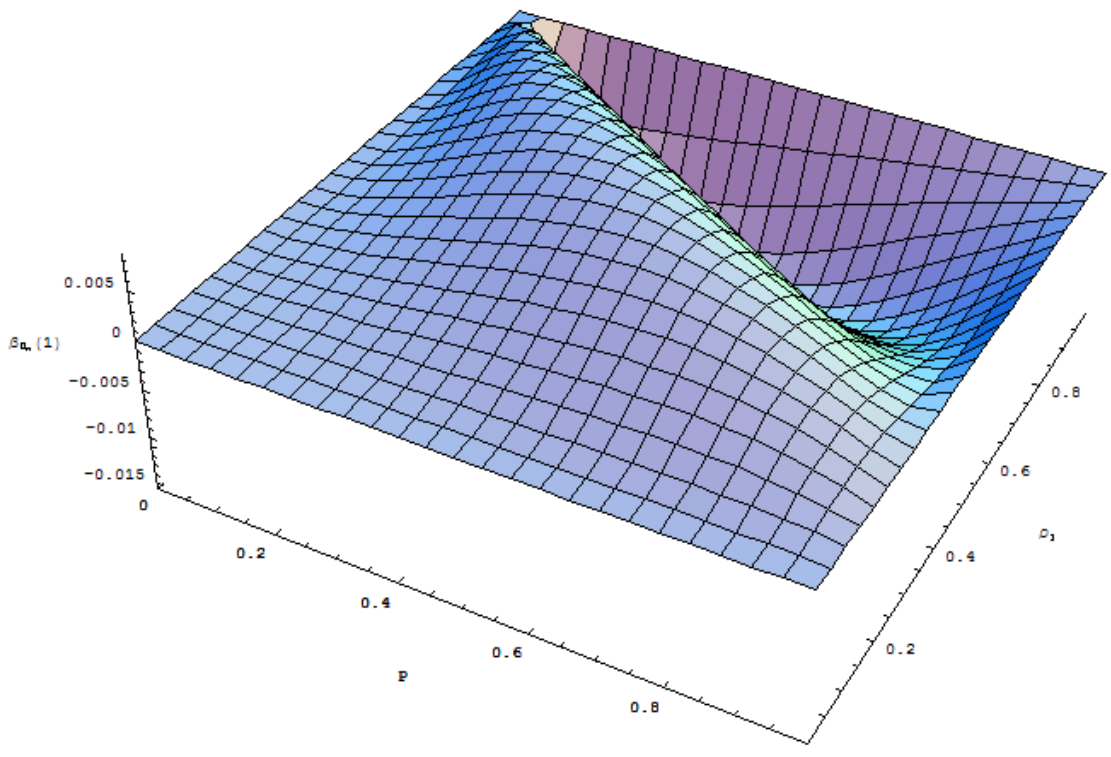

Figure 19: Modified $\beta_{D_{n}}(1)$ as a function of $P$ and $\rho_{1}$

approximations in [25] are obtained by letting the renewal-interval distribution of the departure process from a single-server queue be a convex combination of the renewal-interval distributions from the two basic methods. In this section, we use a similar approach to develop a hybrid approximation for the departure process at the "Wait for release" buffer.

In previous sections, we studied the modified departure process by the stationaryinterval method. The corresponding probability density function $f_{D_{n}}^{S I}\left(d_{n}\right)$ was found in section 4 . Using the reasoning in [25], we now describe the asymptotic method approximation for the departure process at the "Wait for release" buffer. Let $D(t), A(t), Q(t)$ and $R(t)$ represent the number of departures at the "Wait for release" buffer in $[0, t]$, the number of arrivals at "Queue 1 " in $[0, t]$, the number of customers in "Queue 1" and "Station 1 " at time $t$ and the number of customers in the "Wait for release" buffer at time $t$. Clearly, these random variables are related by

$$
D(t)=A(t)-Q(t)+Q(0)-R(t)+R(0) \text { for } t \geq 0
$$

As stated in [25], $Q(t)$ converges in distribution to a proper limit as $t \rightarrow \infty$. The same applies for $R(t)$. If we define $R$ as the number of customers in the "Wait for release" buffer in steady state, $R(t)$ converges to $R$ in distribution as $t \rightarrow \infty$ with $E[R]=\lambda \int_{0}^{L T 1}\left(L T 1-c t_{1}\right) f_{C T_{1}}\left(c t_{1}\right) d c t_{1}<\infty$. Consequently, just 


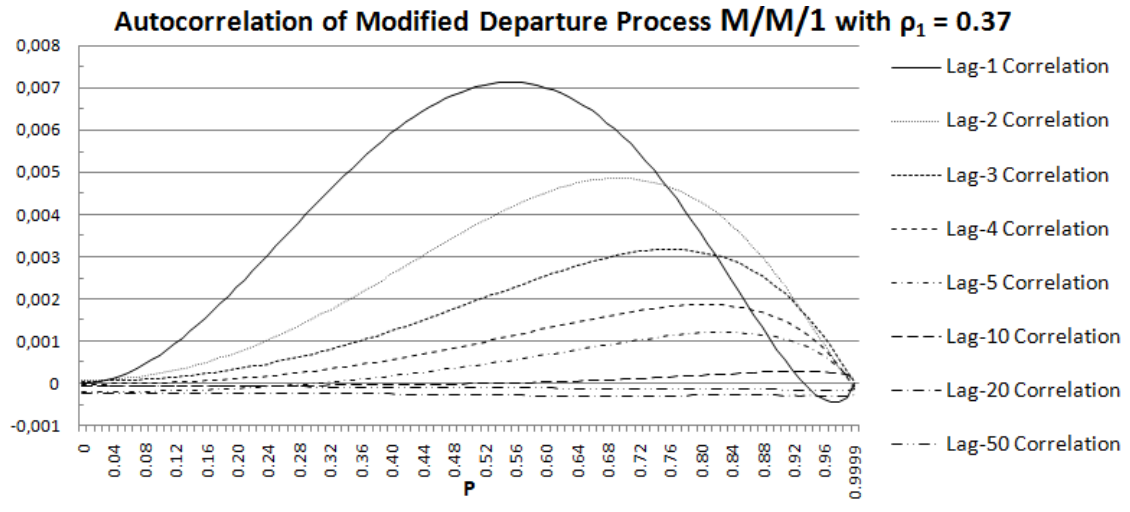

Figure 20: $\beta_{D_{n}}(\tau)$ as a function of $P$ for $\tau=1,2,3,4,5,10,20$ and 50

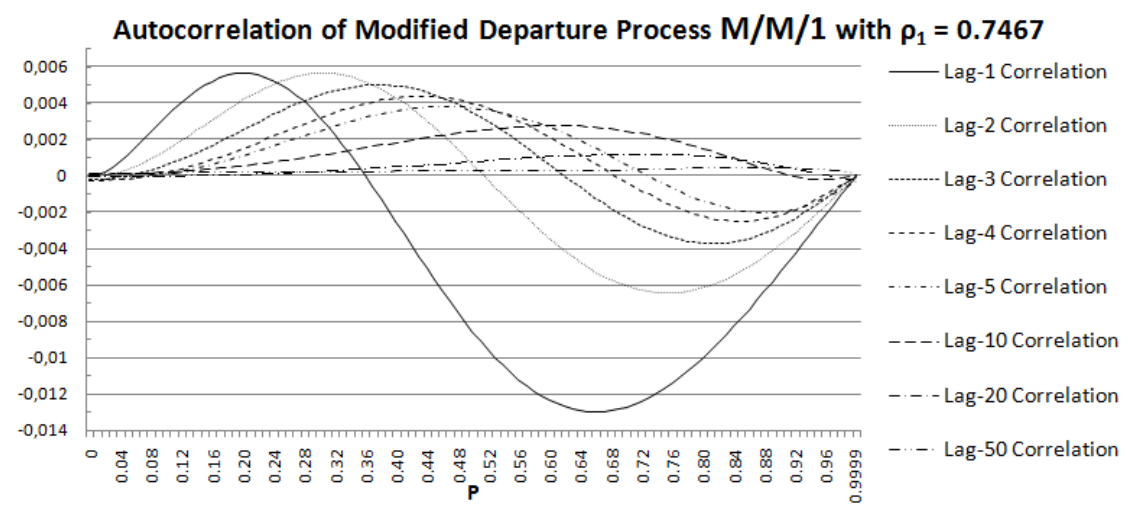

Figure 21: $\beta_{D_{n}}(\tau)$ as a function of $P$ for $\tau=1,2,3,4,5,10,20$ and 50

like $Q(t), R(t)$ remains stochastically bounded:

$$
\sup _{t \geq 0} E\left[|R(t)-R(0)|^{k}\right]<\infty \text { for all } k
$$

Given that $A(t)$ diverges to $+\infty$ while $Q(t)$ and $R(t)$ are converging in distribution to a proper limit as $t \rightarrow \infty$, the distribution of $D(t)$ for large $t$ is very close (relative to $t$ ) to the distribution of $A(t)$. The asymptotic-method approximation for the departure process coincides with the asymptotic method approximation for the arrival process. Because the arrival process is a renewal process with renewal-interval distribution $f_{A_{n}}\left(a_{n}\right)=\lambda e^{-\lambda a_{n}}$, the asymptotic method approximation yields $f_{D_{n}}^{A}\left(d_{n}\right)=\lambda e^{-\lambda d_{n}}$.

One way to develop refined approximations, is to look for convex combina- 


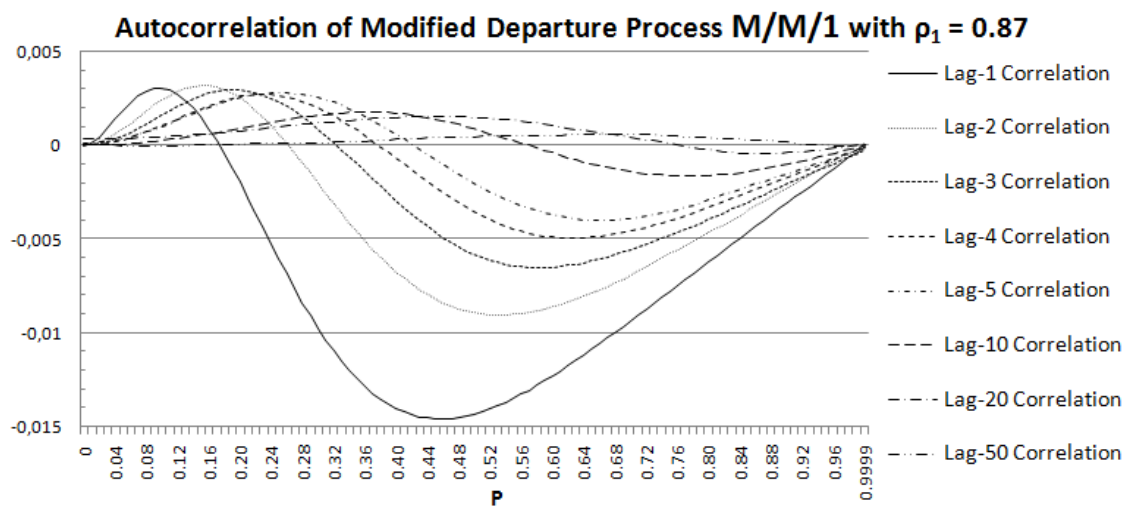

Figure 22: $\beta_{D_{n}}(\tau)$ as a function of $P$ for $\tau=1,2,3,4,5,10,20$ and 50

tions of the interval distributions from the two basic methods:

$$
f_{D_{n}}\left(d_{n}\right)=x f_{D_{n}}^{A}\left(d_{n}\right)+(1-x) f_{D_{n}}^{S I}\left(d_{n}\right)
$$

where $x$ is a weighting function with $0 \leq x \leq 1$. Since we are primarily interested in the departure process at the "Wait for release" buffer as the arrival process at the second workstation, which leads to a criterion involving the queue length at the second station, we should anticipate that the weighting function might involve the utilization of this second station as discussed in [25]. For example, if the second station is in heavy traffic but the first is not ( $\rho_{2}$ is close to 1 but $\rho_{1}$ is not), then the heavy-traffic behavior of the second station is the same as if the first station were not there. Hence, $x$ ought to approach 1 as $\rho_{2} \rightarrow 1$. If both stations are in heavy traffic ( $\rho_{1}$ and $\rho_{2}$ are both close to 1$), x$ should be close to 1 if $\rho_{2}$ is relatively closer to 1 than $\rho_{1}$. Satisfying these heavy-traffic limit theorems, a first hybrid approximation is found by setting $x$ equal to $\rho_{2}$. The evaluation of this refined approximation "Hybrid 1" compares the resulting values of $L T 2$, the 95th percentile of the cycle time at the second workstation, with some simulation results. Appendix D gives the percentage deviations of the computed $L T 2$ values in function of $P$ for different $\left(\rho_{1}, \rho_{2}\right)$ settings as compared with the simulation results. We conclude that the stationary-interval method deserves more weight at higher $P$ values when the system is not characterized by a heavy-traffic limit theorem. A refined approximation is found by setting $x=\rho_{2}\left(1-\rho_{1}^{c}\right)$ with $c=50\left(e^{\rho_{2}-\rho_{1}}\right) e^{-1}\left(1-P^{\left(e^{\rho_{2}-\rho_{1}}\right) e^{-1}}\right)$. This "Hybrid $2 "$ approximation improves the prediction of the system behavior by an average of 0.50 percentage points in $62 \%$ of the settings in Appendix D. The maximum improvement amounts to 1.43 percentage points. In $30 \%$ of the settings, the accuracy decreases by an average of 0.08 percentage points, with a maximum decrease of 0.51 percentage points.

In order to further evaluate the performance of both hybrid approximations, an additional discrete event simulation is runned for 1100 different parameter 
settings: $\rho_{1}, \rho_{2} \in\{\min =0.10 ; \max =0.90 ;$ step $=0.10\} \cup\{0.85\}$ and $P$ $\in\{\min =0.10 ; \max =0.90 ;$ step $=0.10\} \cup\{0.95\}$. In Appendix E, the average percentage deviations of the computed $E[C T 12]$ and $L T 12$ values, as compared with the simulation results, are expressed in percent for the different parameter settings. The bold values indicate settings where the performance measures are underestimated. The underlined values reveal the worst performing regions which are also summerized in Table 3. The latter is completed with the overall average percentage deviations. Consistent with earlier observations,

\begin{tabular}{|l|l|l|l|}
\hline & Average & MAX & $M I N$ \\
\hline$E[C T 12]$ & & & \\
\hline Hybrid 1 & 0.2130 & 2.1762 & -0.7130 \\
\hline Hybrid 2 & 0.1541 & 1.6593 & -0.7131 \\
\hline MAX at & $\rho_{1}=0.90$ & $\rho_{2}=0.90$ & $P=0.60$ \\
\hline MIN at & $\rho_{1}=0.10$ & $\rho_{2}=0.90$ & $P=0.90$ \\
\hline LT12 & & & \\
\hline Hybrid 1 & 0.2148 & 1.4575 & -1.0309 \\
\hline Hybrid 2 & 0.2377 & 0.8431 & -1.0311 \\
\hline MAX at & $\rho_{1}=0.90$ & $\rho_{2}=0.90$ & $P=0.60$ \\
\hline MIN at & $\rho_{1}=0.30$ & $\rho_{2}=0.90$ & $P=0.70$ \\
\hline
\end{tabular}

Table 3: Impact of Intermediate Release Authorizations

we conclude that the worst levels of overestimation occur when a high $P$ value is set in a system which is not characterized by the heavy-traffic limit theorems. A performance gap that reduces significantly by replacing the "Hybrid 1" approximation by the "Hybrid 2" approximation. Unfortunately, by putting more weight on the stationary-interval method in the "Hybrid 2" approximation, the level of underestimation is increasing. These underestimates are especially evident for systems showing a heavy-traffic behavior and are further deteriorating the evaluation of $L T 12$ which is characterized by a high degree of underestimation. However, with a maximum overestimation of $E[C T 12]$ equal to $1.66 \%$ and a maximum underestimation of $L T 12$ equal to $1.03 \%$, we conclude that the "Hybrid 2" approximation achieves a healthy balance between the stationaryinterval method and the asymptotic method.

Important, by proposing a convex combination of the stationary-interval method and the asymptotic method for the departure process at the "Wait for release" buffer in Figure 2, we assure that intermediate release authorizations can only reduce the waiting time in "Queue 2 ". Reminding that the asymptotic departure process at the "Wait for release" buffer in Figure 2 corresponds with the departure process at the first station in Figure 1, this remark is based on the observation that the stationary-interval approximations of $L T 2, E[C T 12]$ or $L T 12$ were always smaller than the correseponding asymptotic method approximations. However, it will be clear that time-phased order releases can never reduce the total cycle time as it may only slow down the system flow. The reduced waiting times in "Queue 2" will be more than compensated for by 
the additional waiting times in the "Wait for release" buffer.

We finish this section with the application of both hybrid approximations to the example computed in section 6. In Table 2, "Approximation 1" and "Approximation 2" respectively correspond to the stationary-interval method and the asymptotic method. If we use the above defined "Hybrid 1" and "Hybrid 2" approximations, we obtain $E[C T 12]$ values equal to 9.47 and 9.44 and LT12 values equal to 19.77 and 19.67. As a high $P$-value $(79.81 \%)$ is set in a system without heavy-traffic behavior $\left(\rho_{1}=0.6, \rho_{2}=0.75\right)$, both hybrid approximations overestimate $E[C T 12]=9.40$ because too much weight is put on the asymptotic method while the "Hybrid 2" approximation underestimates $L T 12=19.74$ because to much weight is put on the stationary-interval method. However, with an overestimation of $E[C T 12]$ by only $0.43 \%$ and underestimation of $L T 12$ by only $0.35 \%$, the "Hybrid 2 " approximation yields a satisfying approximation.

An important observation to make is that time-phased order releases reduce the system variability. By setting $P=80 \%$ in our example, the expected time to flow through the whole system $E$ [CT12] increases by 1.90 time units or $25 \%$ while the required safety time $L T 12-E[C T 12]$, needed to buffer the system variability, reduces by 0.54 time units or $5 \%$. Because originally $60 \%$ safety time was included, the quoted lead time $L T 12$ only increases by 1.36 time units or $7 \%$ when intermediate release authorizations are set. Reproducing the evolution

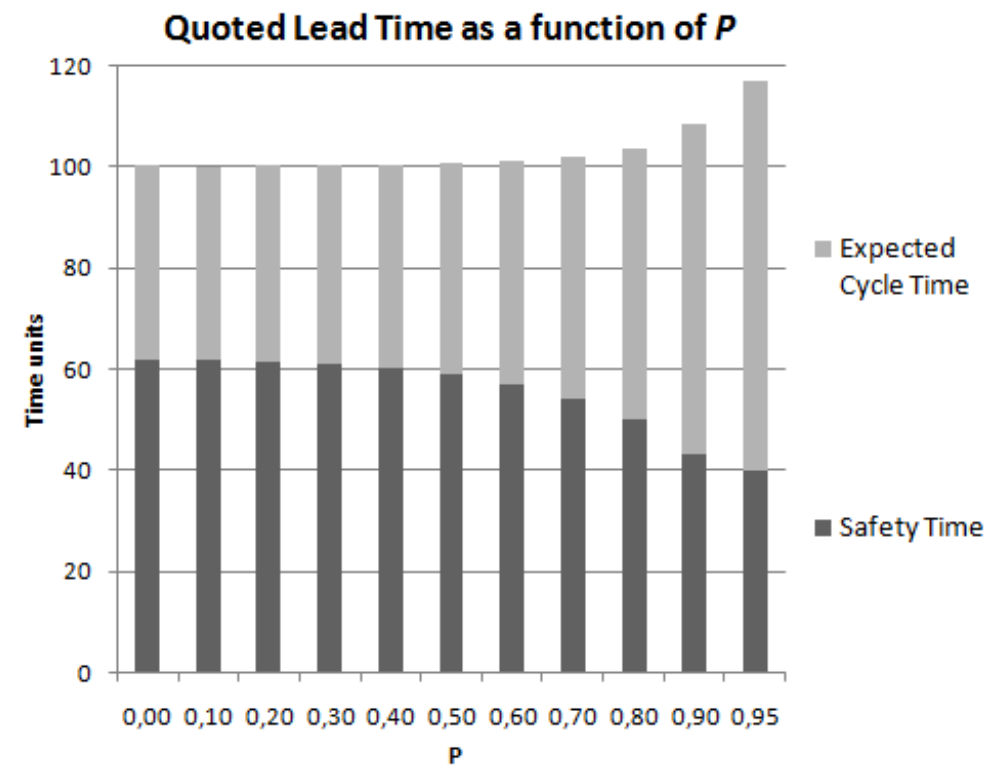

Figure 23: Time-phased order releases reduce system variability

of the average $L T 12, E[C T 12]$ and $L T 12-E[C T 12]$ values over all simulated $\left(\rho_{1}, \rho_{2}\right)$ settings in function of $P$, Figure 23 shows a similar reduction of the 
system variability. On average, a $P$-value equal to $80 \%$ will equate the required safety time $L T 12-E[C T 12]$ with the expected cycle time $E[C T 12]$ and increase the quoted lead time $L T 12$ by $3.5 \%$. The required safety time decreases from 62.1 time units to 51.1 time units, a decrease of $18 \%$. The expected cycle time increases from 37.9 time units to 52.4 time units, an increase of $38 \%$. Furter research should study the influence of specific parameter settings.

\section{Conclusion}

In this paper, we analyzed the consequences of time-phased order releases by introducing them in an open queueing network composed of two M/M/1 stations. The core of the analysis was focused on the modified flow variability specified by the departure process at the fist station.

First, we approximated the modfied departure process by a renewal process using the stationary-interval method. It showed how the flow variability reduces by slowing down customers with short cycle times at the first station. A downward trend that fades away when too much safety time is allocated, when idle time periods dominate the departure process at low utilization levels or when long waiting times at the first workstation are dominating the system behavior at high utilization levels. We also observed that the modified departure process is only a second-order stationary process with negligible autocorrelation.

Secondly, we studied the asymptotic method approximation of the modified departure process in order to better take into account he dependency among successive departure intervals. It allowed us to develop some refined approximations of the modified departure process as a renewal process. By assuming a convex combination of both approximation methods, we respected the characteristic that intermediate release authorizations can only reduce the waiting times at the second station. These new expressions allowed us to quantify the increase of the expected time to flow through the whole system, the corresponding safety time needed to meet a given service level... As a result, we quantified the absorption of system variabilty into allocated time buffers.

It will be clear that a lot of further research is needed to drop all simplifying assumptions in this initial effort to model time-phased order releases. For example, in order to study the impact of different variability levels in the arrival and service process, a network with two G/G/1 stations should be modelled. Even the analysis of a network composed of three successive $M / M / 1$ stations needs additional study. The reason has to be searched in the fact that the consequences of time-phased order releases at the third station will not only depend on the cycle time at the second station. We are convinced that this research is absolutely necessary to increase the usefulness of queueing network models in advanced planning systems for contemporary supply chains. 


\section{References}

[1] Bergamaschi D., R. Cigolini, M. Perona, A. Portioli. 1997. Order review and release strategies in a job shop environment: a review and a classiffication. International Journal.of Production. Research. 35(2). 399-420.

[2] Bracewell, R. 1999. The Fourier Transform and Its Applications, 3rd edition. McGraw-Hill. New York.

[3] Burke, P.J. 1956. The output of a queueing system. Operations Research. 4. 699-704.

[4] Buzacott, J.A. 1989. Queueing models of Kanban and MRP controlled production systems. Engineering Costs and Production Economics. 17. 3-20.

[5] Daley, D.J. 1968. The correlation structure of the output process for some single server queueing systems. Annals of Mathematical Statistics. 39(3). 1007-1019.

[6] Daley, D. J. 1976. Queueing output process. Advances in Applied Probability. 8. 395-415.

[7] Elhafsi, M. 2002. Optimal leadtimes planning in serial production systems with earliness and tardiness costs. IIE Transactions. 34(3). 233-243.

[8] Enns, S.T. 2001. MRP performance effects due to lot size and planned lead time settings. International Journal of Production Research. 39(3). 461-480.

[9] Ferng, H.W., J.F. Chang. 2001. Departure process of BMAP/G/1 queues. Queueing Systems. 39. 109-135.

[10] Girish, M.K., J.Q. Hu. 2001. Approximations for the departure process of the $G / G / 1$ queue with Markov-modulated arrivals. European Journal of Operations Research. 134. 540-556.

[11] Gong, L., T. de Kok, J. Ding. 1994. Optimal Leadtimes Planning in serial Production System. Management Science. 40(5). 629-632.

[12] Hasan, C.N, M.L. Spearman. 1999. Optimal material release times in stochastic production environments. International Journal of Production Research. 37(6). 1201-1216.

[13] Hopp, W.J., M.L. Spearman. 2007. Factory Physics. Irwin/ McGraw-Hill. New York.

[14] Kanet, J.J., D.P. Christy. 1984. Manufacturing systems with forbidden early order departure. International Journal of Operations Research. 22(1). 41-50.

[15] Kleinrock, L. 1975. Queueing Systems, Volume 1: Theory. John Wiley \& Sons. New York. 
[16] Koh, S.C.L., S.M. Saad. 2003. MRP-controlled manufacturing environment disturbed by uncertainty. Robotics and Computer Integrated Manufacturing. 19. 157-171.

[17] Lim, S.Y., S. Hur, S.J. Noh. 2006. Departure process of a single server queueing system with Markov renewal input and general service time distribution. Computers \& Industrial Engineering. 51. 519-525.

[18] Livny, M., B. Melamed, A.K. Tsiolis. 1993. The impact of autocorrelation on queueing systems. Management Science. 39(3). 322-339.

[19] Pahl, J., S. Voß, D.L. Woodruff. 2007. Production planning with load dependent lead times: an update of research. Annals of Operations Research. 153. $297-345$.

[20] Song, D., C. Hicks, C. F. Earl. 2001. Setting planned job release times in stochastic assembly systems with resource constraints. International Journal of Production Research. 39(6). 1289-1301.

[21] Vandaele, N.J., M.R. Lambrecht. 2003. Reflections on the use of stochastic manufacturing models for planning decisions. J.G. Shanthikumar, D. D. Yao, W. H. M. Zijm, eds. Stochastic Modeling and Optimization of Manufacturing Systems and Supply Chains. Kluwer Academic Publishers. Dordrecht, The Netherlands. 53-85.

[22] Vollmann, T. E., W. L. Berry, D. C. Whybark, F. R. Jacobs. 2005. Manufacturing Planning and Control Systems for Supply Chain Management. McGraw Hill. New York.

[23] Wellborn, C. 2008. Strengthening Supply Chains, Lead-Time confidence intervals address issues of inherent variability as supply chains become more complex. OR/MS Today. 35(3).

[24] Whitt, W. 1982. Approximating a Point Process by a Renewal Process, I: Two Basic Methods. Operations Research. 30(1). 124-147.

[25] Whitt, W. 1984. Approximations for departure processes and queues in series. Naval Research Logistics Quarterly. 31. 499-521.

[26] Wisner, J.D. 1995. A review of the order release policy research. International Journal of Operations \& Production Management. 15(6). 25-40.

[27] Yano, C.A. 1987. Setting planned leadtimes in serial production systems with tardiness costs. Management science. (33)11. 95-106. 


\section{A Proof of the Sifting Property}

A proof of the sifting property stating that:

$$
\int_{-\infty}^{\infty} \delta\left(t-t_{0}\right) u(t) d t=u\left(t_{0}\right)
$$

can be found in [2]. If we define $\delta(t)$ as $\lim _{\tau \rightarrow 0} d_{\tau}(t)$ with $d_{\tau}(t)=1 / 2 \tau$ for $|t|<\tau$ and $d_{\tau}(t)=0$ for $|t| \geq \tau$, then:

$$
\int_{-\infty}^{\infty} \delta\left(t-t_{0}\right) u(t) d t=\lim _{\tau \rightarrow 0} \int_{-\infty}^{\infty} d_{\tau}\left(t-t_{0}\right) u(t) d t
$$

Using the definition of $d_{\tau}(t)$ and the first mean-value theorem for integration:

$$
\begin{aligned}
\int_{-\infty}^{\infty} \delta\left(t-t_{0}\right) u(t) d t & =\lim _{\tau \rightarrow 0} \frac{1}{2 \tau} \int_{t_{0}-\tau}^{t_{0}+\tau} u(t) d t \\
& =\lim _{\tau \rightarrow 0} \frac{1}{2 \tau}(2 \tau) u\left(t^{*}\right) \\
& =\lim _{\tau \rightarrow 0} u\left(t^{*}\right)
\end{aligned}
$$

with $t_{0}-\tau<t^{*}<t_{0}+\tau$. Because $t^{*} \rightarrow t_{0}$ when $\tau \rightarrow 0$ :

$$
\int_{-\infty}^{\infty} \delta\left(t-t_{0}\right) u(t) d t=u\left(t_{0}\right)
$$

\section{B Extended tree to compute lag-1 correlation}

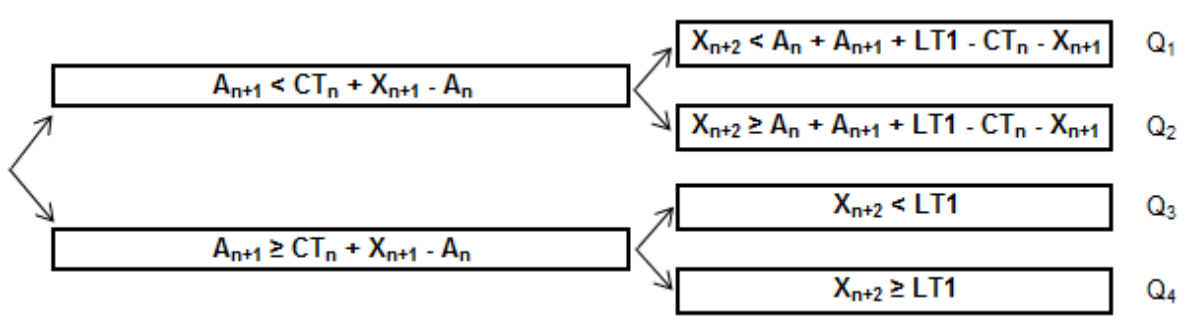

Sub-events of $C_{1}$ 


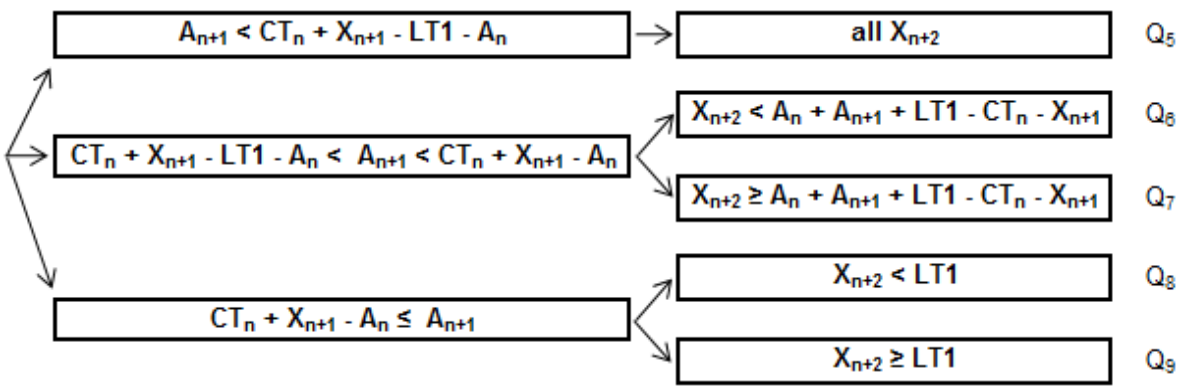

Sub-events of $C_{2}$

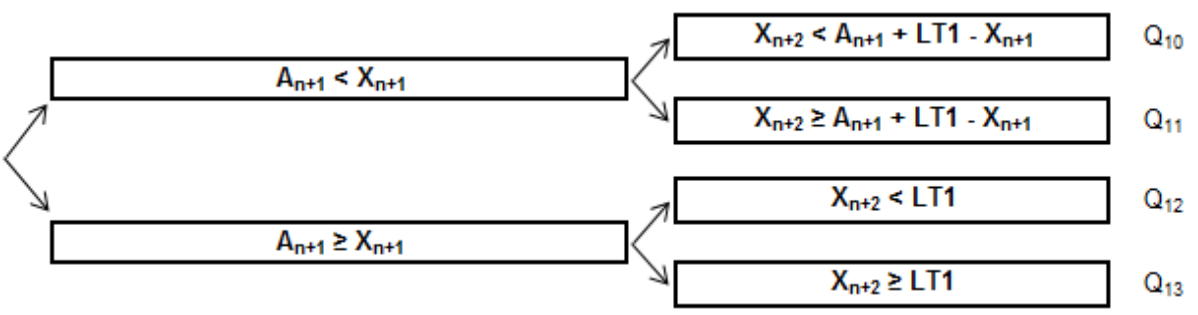

Sub-events of $C_{3}$

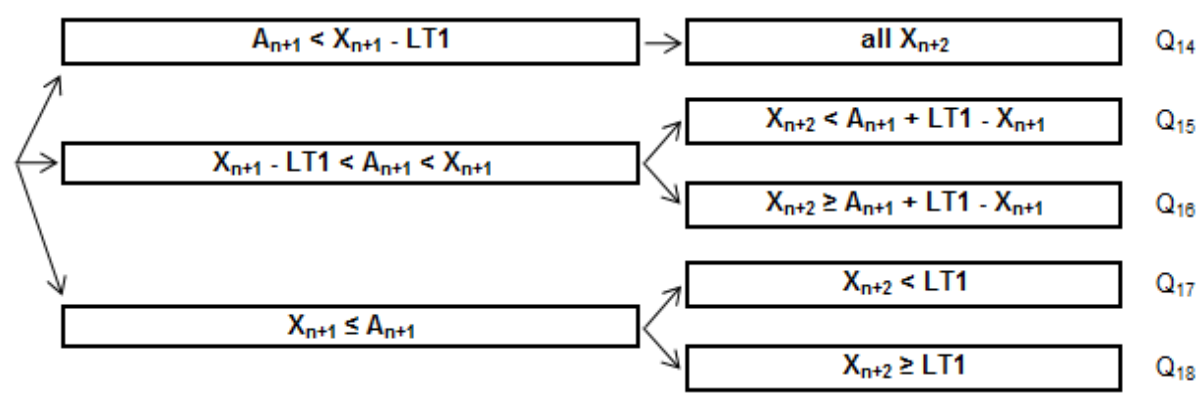

Sub-events of $C_{4}$

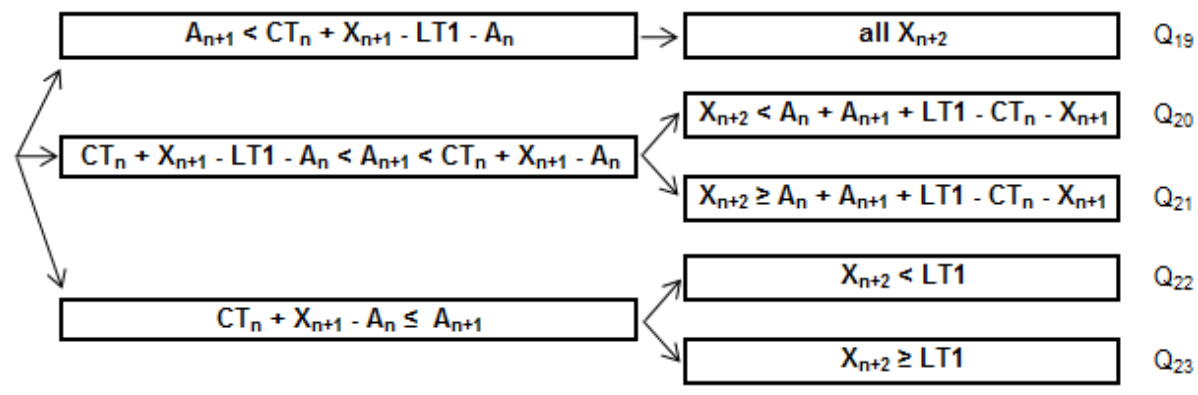

Sub-events of $C_{5}$ 


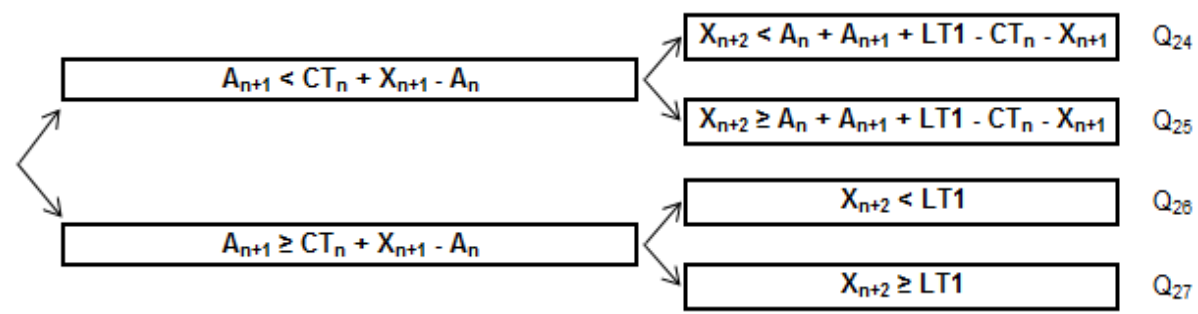

Sub-events of $C_{6}$

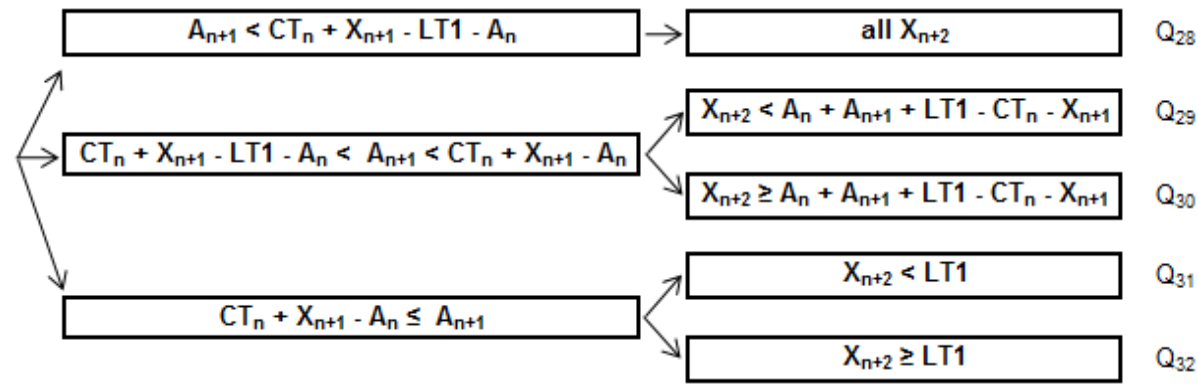

Sub-events of $C_{7}$

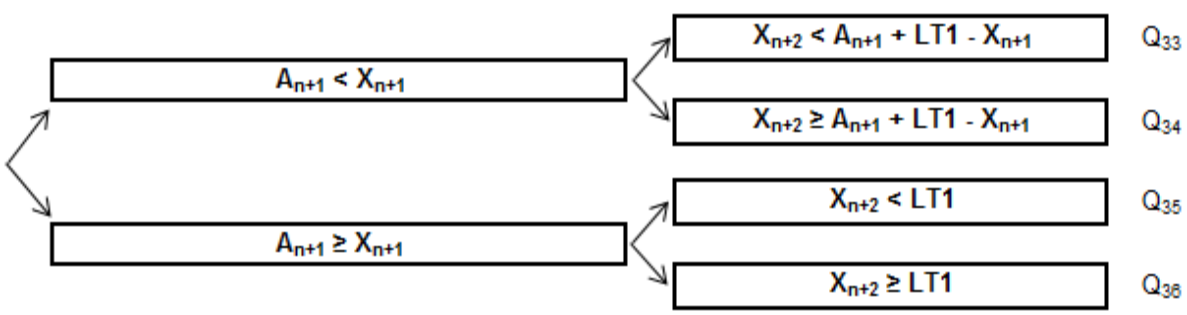

Sub-events of $C_{8}$

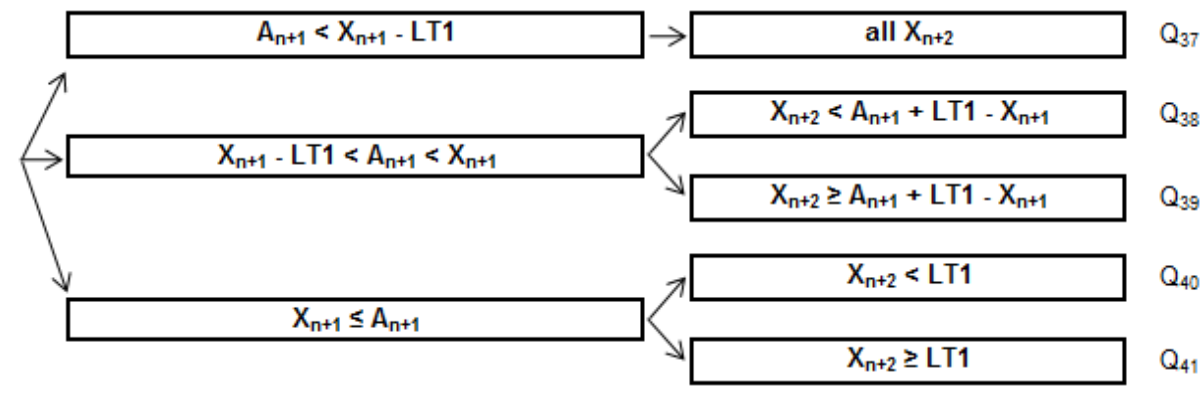

Sub-events of $C_{9}$ 


\section{Quantification of $\left(D_{n}, D_{n+1}\right)$ for sub-events $Q_{i}$}

\begin{tabular}{|c|c|c|c|}
\hline $\mathbf{C}_{i}$ & $\mathbf{D}_{n}$ & $\mathbf{Q}_{\mathrm{i}}$ & $\mathbf{D}_{n+1}$ \\
\hline$C_{1}$ & $A_{n}$ & $\begin{array}{l}Q_{1} \\
Q_{2} \\
Q_{3} \\
Q_{4}\end{array}$ & $\begin{array}{c}A_{n+1} \\
C T_{n}+X_{n+1}+X_{n+2}-A_{n}-L T 1 \\
A_{n+1} \\
A_{n+1}+X_{n+2}-L T 1\end{array}$ \\
\hline$C_{2}$ & $C T_{n}+X_{n+1}-L T 1$ & $\begin{array}{l}Q_{5} \\
Q_{6} \\
Q_{7} \\
Q_{8} \\
Q_{9}\end{array}$ & $\begin{array}{c}X_{n+2} \\
A_{n}+A_{n+1}+L T 1-C T_{n}-X_{n+1} \\
X_{n+2} \\
A_{n}+A_{n+1}+L T 1-C T_{n}-X_{n+1} \\
A_{n}+A_{n+1}+X_{n+2}-C T_{n}-X_{n+1}\end{array}$ \\
\hline$C_{3}$ & $A_{n}$ & $\begin{array}{l}Q_{10} \\
Q_{11} \\
Q_{12} \\
Q_{13}\end{array}$ & $\begin{array}{c}A_{n+1} \\
X_{n+1}+X_{n+2}-L T 1 \\
A_{n+1} \\
A_{n+1}+X_{n+2}-L T 1\end{array}$ \\
\hline$C_{4}$ & $A_{n}+X_{n+1}-L T 1$ & $\begin{array}{l}Q_{14} \\
Q_{15} \\
Q_{16} \\
Q_{17} \\
Q_{18}\end{array}$ & $\begin{array}{c}X_{n+2} \\
A_{n+1}+L T 1-X_{n+1} \\
X_{n+2} \\
A_{n+1}+L T 1-X_{n+1} \\
A_{n+1}+X_{n+2}-X_{n+1}\end{array}$ \\
\hline$C_{5}$ & $X_{n+1}$ & $\begin{array}{l}Q_{19} \\
Q_{20} \\
Q_{21} \\
Q_{22} \\
Q_{23} \\
\end{array}$ & $\begin{array}{c}X_{n+2} \\
A_{n}+A_{n+1}+L T 1-C T_{n}-X_{n+1} \\
X_{n+2} \\
A_{n}+A_{n+1}+L T 1-C T_{n}-X_{n+1} \\
A_{n}+A_{n+1}+X_{n+2}-C T_{n}-X_{n+1}\end{array}$ \\
\hline$C_{6}$ & $A_{n}+L T 1-C T_{n}$ & $\begin{array}{l}Q_{24} \\
Q_{25} \\
Q_{26} \\
Q_{27}\end{array}$ & $\begin{array}{c}A_{n+1} \\
C T_{n}+X_{n+1}+X_{n+2}-A_{n}-L T 1 \\
A_{n+1} \\
A_{n+1}+X_{n+2}-L T 1\end{array}$ \\
\hline$C_{7}$ & $X_{n+1}$ & $\begin{array}{l}Q_{28} \\
Q_{29} \\
Q_{30} \\
Q_{31} \\
Q_{32}\end{array}$ & $\begin{aligned} & X_{n+2} \\
A_{n}+A_{n+1}+ & L T 1-C T_{n}-X_{n+1} \\
& X_{n+2} \\
A_{n}+A_{n+1}+ & L T 1-C T_{n}-X_{n+1} \\
A_{n}+A_{n+1}+ & X_{n+2}-C T_{n}-X_{n+1}\end{aligned}$ \\
\hline$C_{8}$ & $A_{n}+L T 1-C T_{n}$ & $\begin{array}{l}Q_{33} \\
Q_{34} \\
Q_{35} \\
Q_{36}\end{array}$ & $\begin{array}{c}A_{n+1} \\
X_{n+1}+X_{n+2}-L T 1 \\
A_{n+1} \\
A_{n+1}+X_{n+2}-L T 1\end{array}$ \\
\hline$C_{9}$ & $A_{n}+X_{n+1}-C T_{n}$ & $\begin{array}{l}Q_{37} \\
Q_{38} \\
Q_{39} \\
Q_{40} \\
Q_{41}\end{array}$ & $\begin{aligned} & X_{n+2} \\
A_{n+1}+ & L T 1-X_{n+1} \\
& X_{n+2} \\
A_{n+1}+ & L T 1-X_{n+1} \\
A_{n+1}+ & X_{n+2}-X_{n+1}\end{aligned}$ \\
\hline
\end{tabular}




\section{Selection of weighting function}
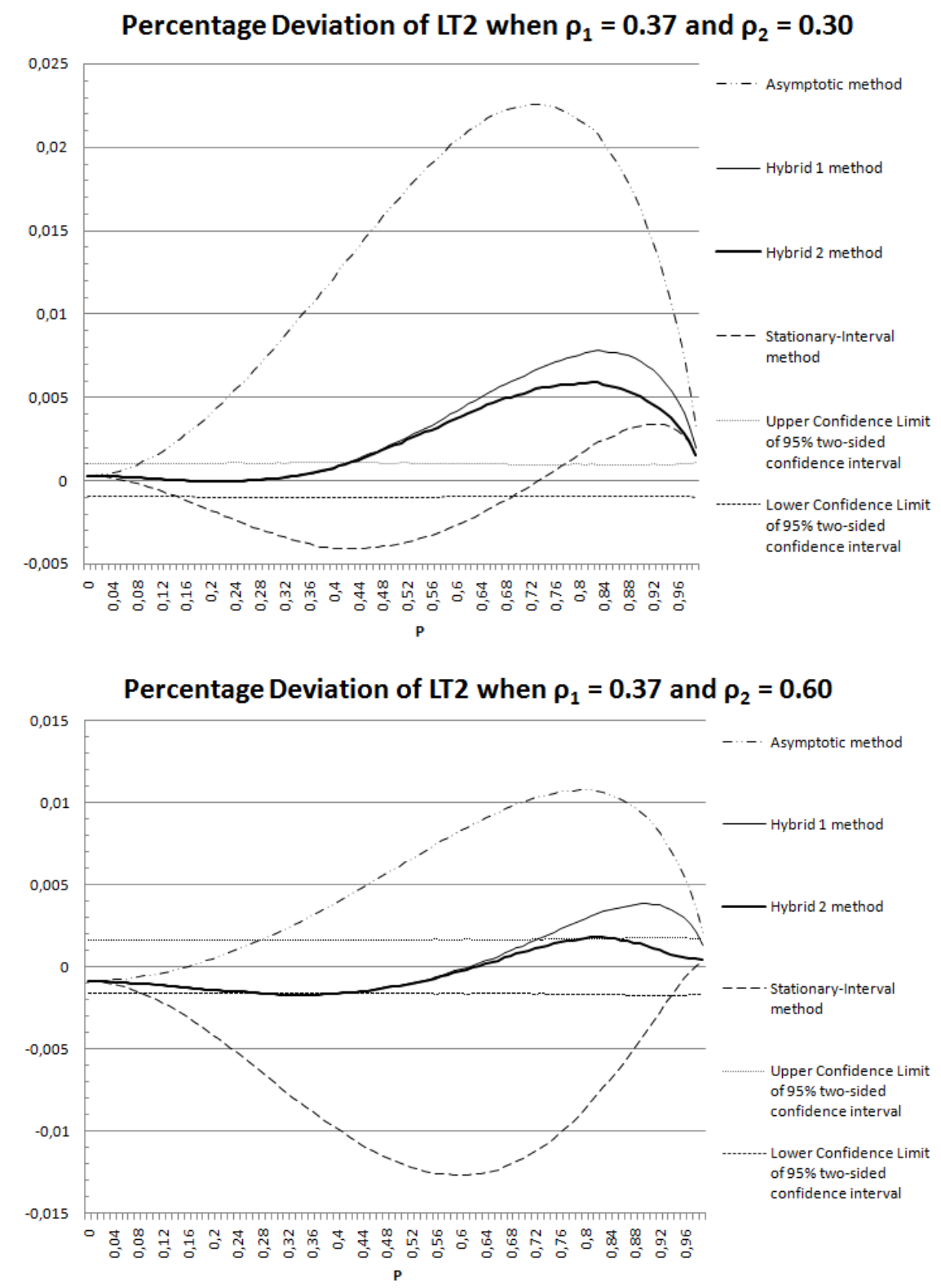

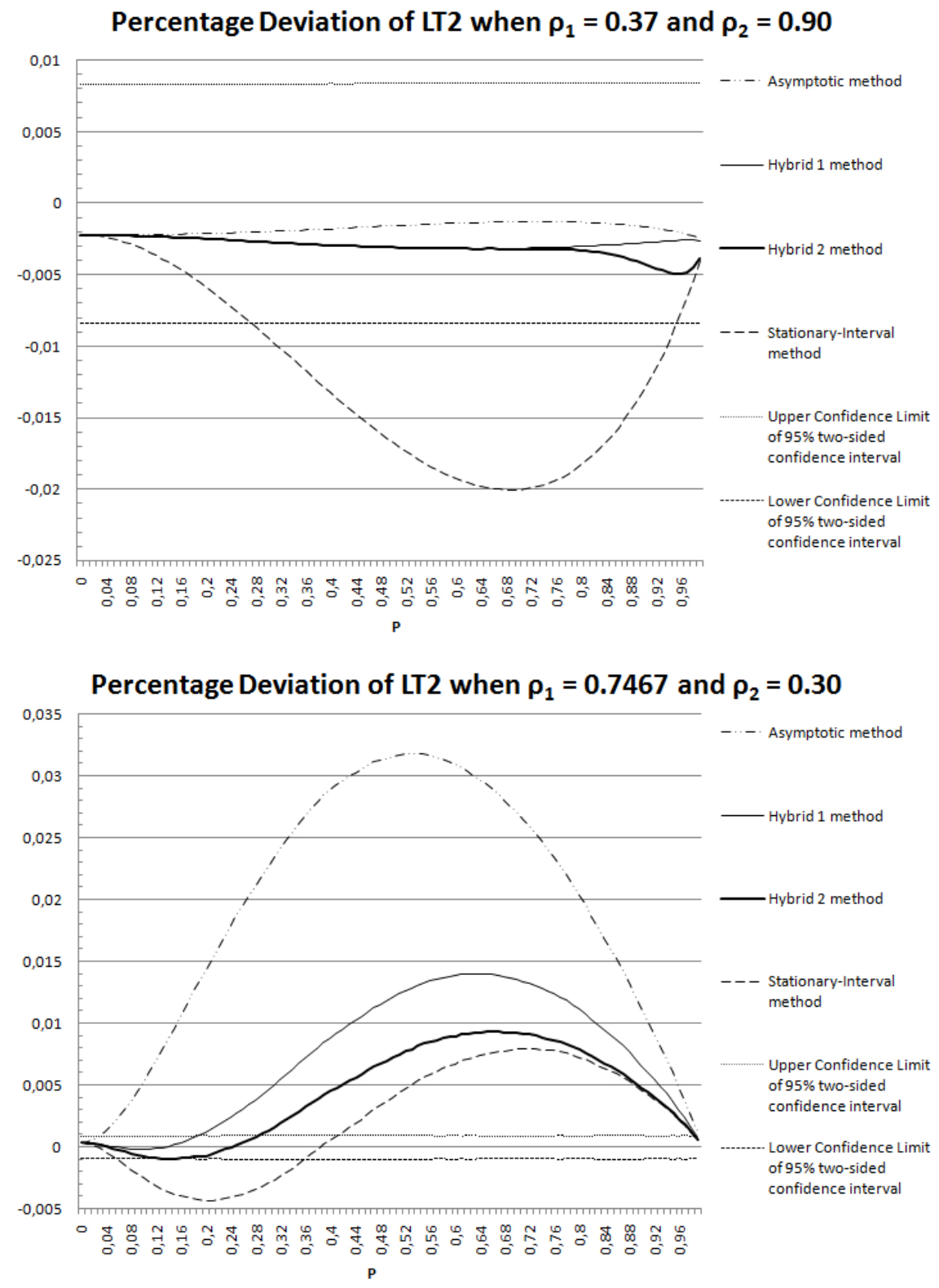


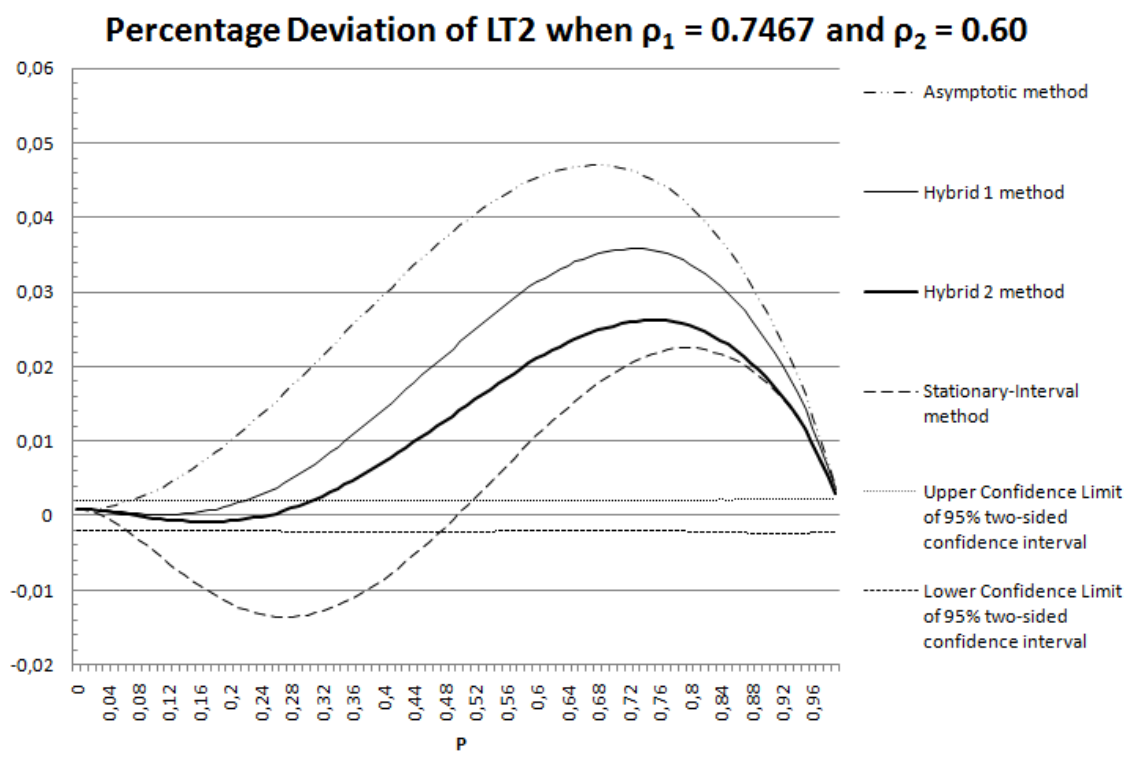

Percentage Deviation of LT2 when $\rho_{1}=\mathbf{0 . 7 4 6 7}$ and $\rho_{2}=\mathbf{0 . 9 0}$

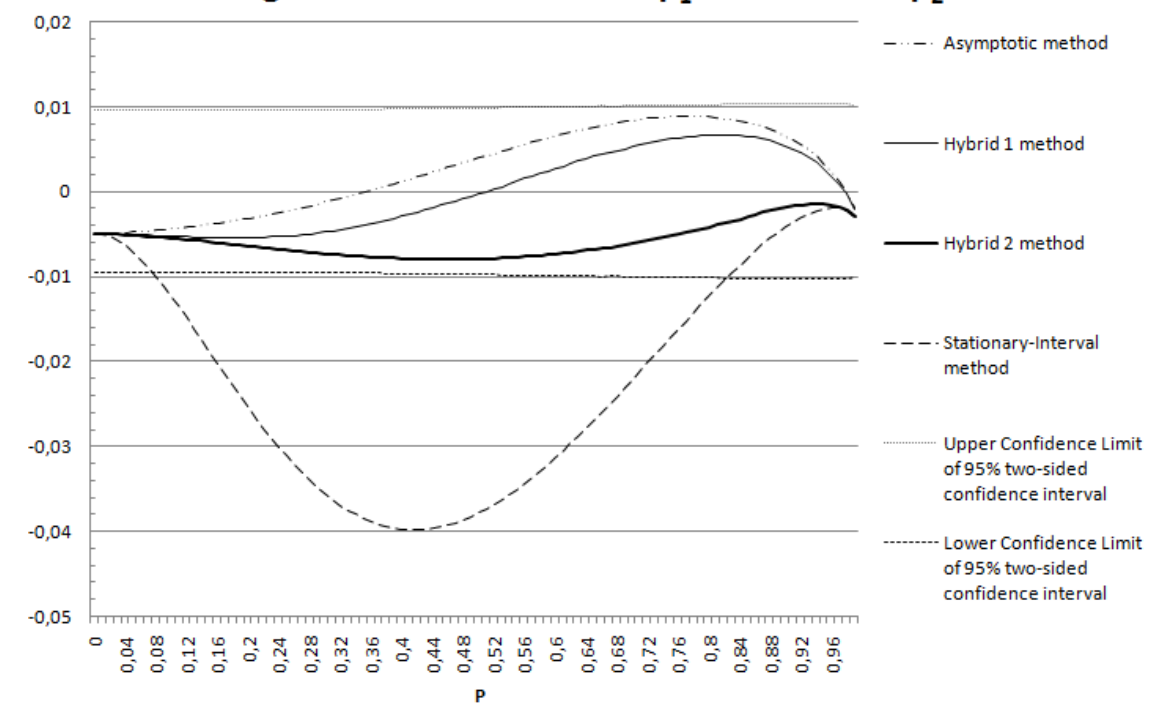




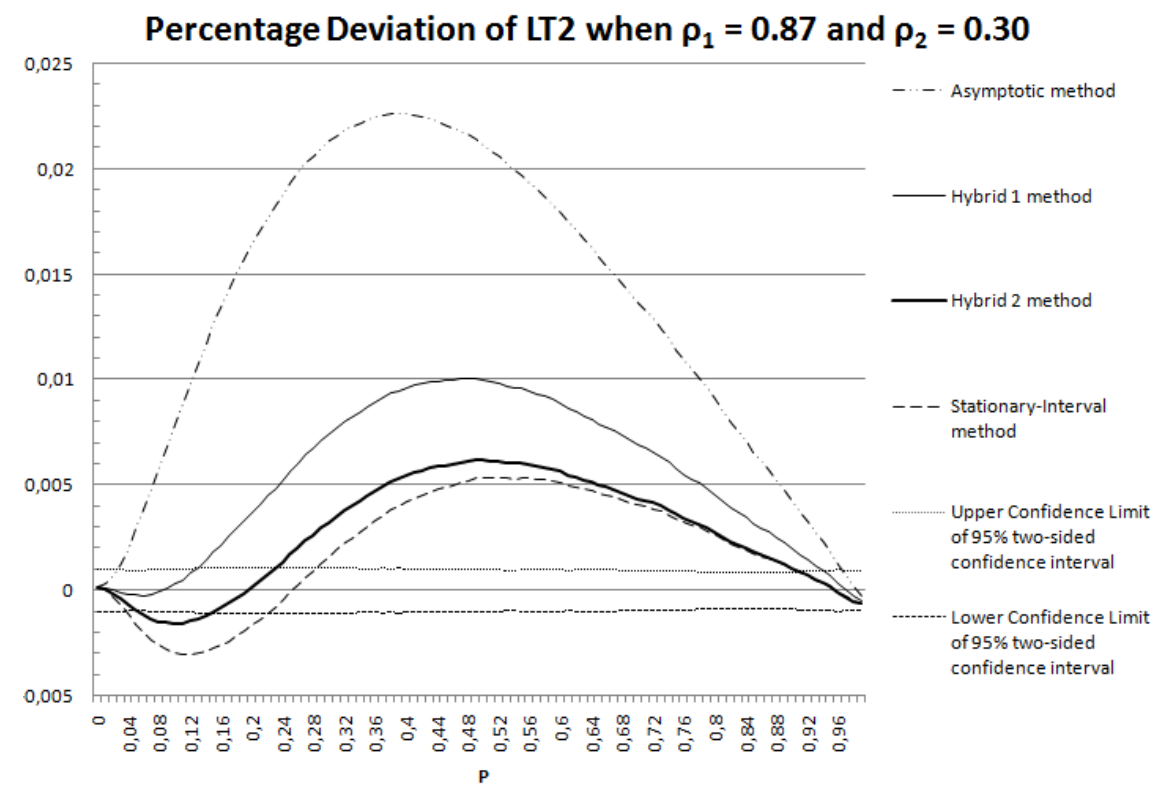

Percentage Deviation of LT2 when $\rho_{1}=\mathbf{0 . 8 7}$ and $\rho_{2}=\mathbf{0 . 6 0}$

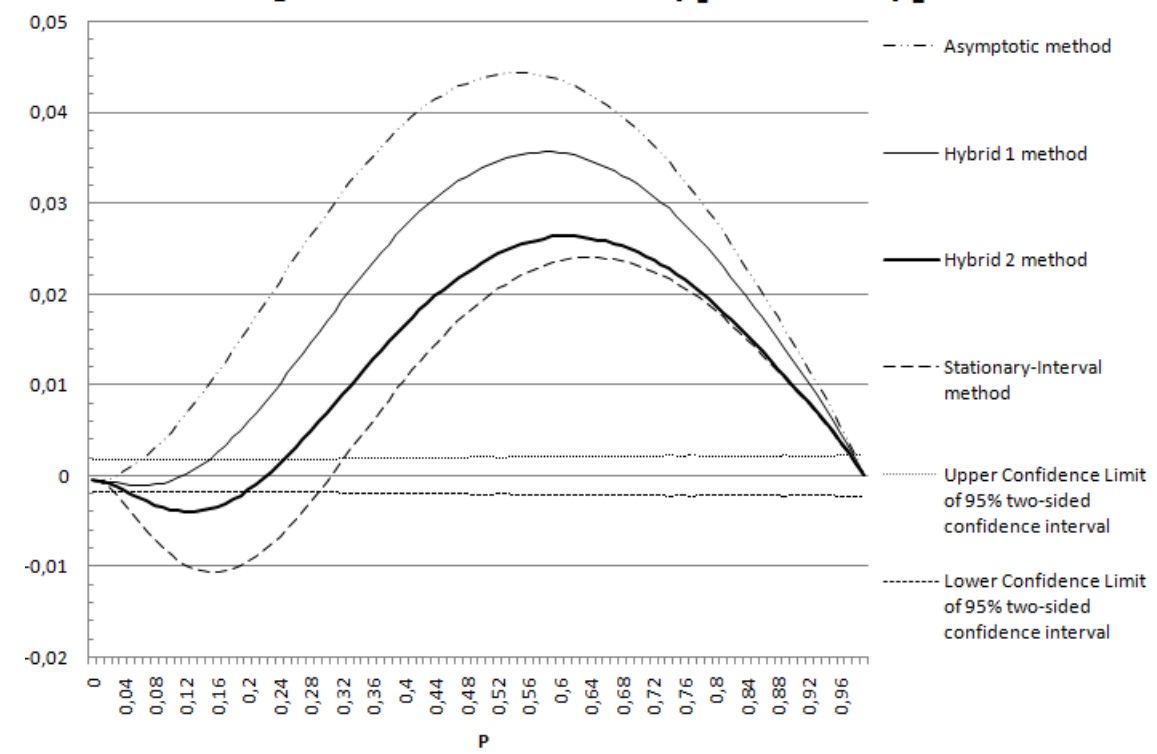




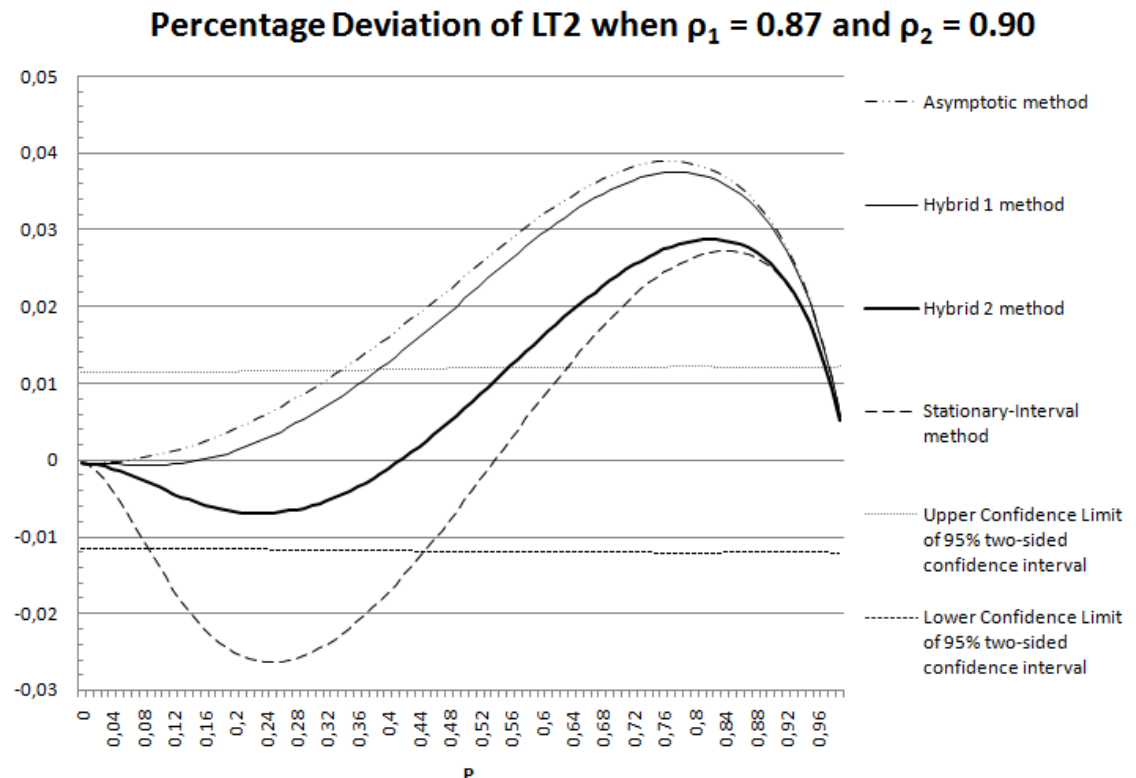




\section{E Evaluation of hybrid approximations}

\begin{tabular}{|c|c|c|c|c|c|c|}
\hline \multirow{3}{*}{$\begin{array}{c}\rho 1 \\
{[0.00: 0.20[}\end{array}$} & \multirow[b]{2}{*}{$\rho 2$} & \multicolumn{5}{|c|}{$P$} \\
\hline & & {$[0.00 ; 0.20[$} & {$[0.20 ; 0.40$} & {$[0.40 ; 0.60[$} & {$[0.60 ; 0.80[$} & {$[0.80 ; 1.00]$} \\
\hline & {$[0.00 ; 0.20[$} & 0.0126 & 0.0054 & 0.0073 & 0.0091 & 0.0118 \\
\hline$[0.00 ; 0.20[$ & {$[0.20 ; 0.40[$} & 0.0240 & 0.0176 & 0.0168 & 0.0152 & 0.0170 \\
\hline$[0.00 ; 0.20[$ & {$[0.40 ; 0.60[$} & 0.0226 & 0.0166 & 0.0507 & 0.0289 & 0.0298 \\
\hline$[0.00 ; 0.20[$ & {$[0.60 ; 0.80[$} & 0.0880 & 0.0496 & 0.0410 & 0.0588 & 0.0531 \\
\hline$[0.00 ; 0.20[$ & {$[0.80 ; 1.00]$} & 0.2349 & 0.1720 & 0.1297 & 0.1165 & $\underline{0.1693}$ \\
\hline$[0.20 ; 0.40[$ & {$[0.00 ; 0.20[$} & 0.0170 & 0.0216 & 0.0115 & 0.0248 & 0.0192 \\
\hline$[0.20 ; 0.40[$ & {$[0.20 ; 0.40[$} & 0.0109 & 0.0233 & 0.0362 & 0.0781 & 0.0769 \\
\hline$[0.20 ; 0.40[$ & {$[0.40 ; 0.60[$} & 0.0257 & 0.0435 & 0.0311 & 0.0981 & 0.1173 \\
\hline$[0.20 ; 0.40[$ & {$[0.60 ; 0.80[$} & 0.0591 & 0.0542 & 0.0707 & 0.0984 & 0.0731 \\
\hline$[0.20 ; 0.40[$ & {$[0.80 ; 1.00]$} & 0.1724 & 0.1973 & 0.1064 & 0.1749 & 0.1686 \\
\hline$[0.40 ; 0.60[$ & {$[0.00 ; 0.20[$} & 0.0333 & 0.0375 & 0.0131 & 0.0115 & 0.0119 \\
\hline$[0.40 ; 0.60[$ & {$[0.20 ; 0.40[$} & 0.0213 & 0.0317 & 0.0916 & 0.1252 & 0.0945 \\
\hline$[0.40 ; 0.60[$ & {$[0.40 ; 0.60[$} & 0.0248 & 0.0397 & 0.1743 & 0.3430 & 0.2548 \\
\hline$[0.40 ; 0.60[$ & {$[0.60 ; 0.80[$} & 0.0404 & 0.0781 & 0.1733 & 0.3976 & 0.3295 \\
\hline$[0.40 ; 0.60[$ & {$[0.80 ; 1.00]$} & 0.0833 & 0.1701 & 0.1797 & 0.2529 & 0.1949 \\
\hline$[0.60 ; 0.80[$ & {$[0.00 ; 0.20[$} & 0.0886 & 0.0394 & 0.0600 & 0.0420 & 0.0197 \\
\hline$[0.60 ; 0.80[$ & {$[0.20 ; 0.40[$} & 0.0447 & 0.0514 & 0.0918 & 0.1108 & 0.0507 \\
\hline$[0.60 ; 0.80[$ & {$[0.40 ; 0.60[$} & 0.0410 & 0.0655 & 0.2883 & 0.4314 & 0.2164 \\
\hline$[0.60 ; 0.80[$ & {$[0.60 ; 0.80[$} & 0.0477 & 0.1231 & 0.4833 & 0.8132 & 0.4996 \\
\hline$[0.60 ; 0.80[$ & {$[0.80 ; 1.00]$} & 0.1804 & 0.1837 & 0.3816 & 0.7882 & 0.5650 \\
\hline$[0.80 ; 1.00]$ & {$[0.00 ; 0.20[$} & 0.1085 & 0.1801 & 0.1306 & 0.0988 & 0.0403 \\
\hline$[0.80 ; 1.00]$ & {$[0.20 ; 0.40[$} & 0.1358 & 0.1153 & 0.0982 & 0.1138 & 0.0279 \\
\hline$[0.80 ; 1.00]$ & {$[0.40 ; 0.60[$} & 0.1854 & 0.1512 & 0.1559 & 0.1747 & 0.0577 \\
\hline$[0.80 ; 1.00]$ & {$[0.60 ; 0.80[$} & 0.0956 & 0.2703 & 0.5496 & 0.5470 & 0.2182 \\
\hline$[0.80 ; 1.00]$ & {$[0.80 ; 1.00]$} & 0.2038 & 0.4270 & 1.0832 & 1.4373 & 0.6641 \\
\hline
\end{tabular}

Percentage Deviation of $E[C T 12]$ when "Hybrid 1" is used (in \%) 


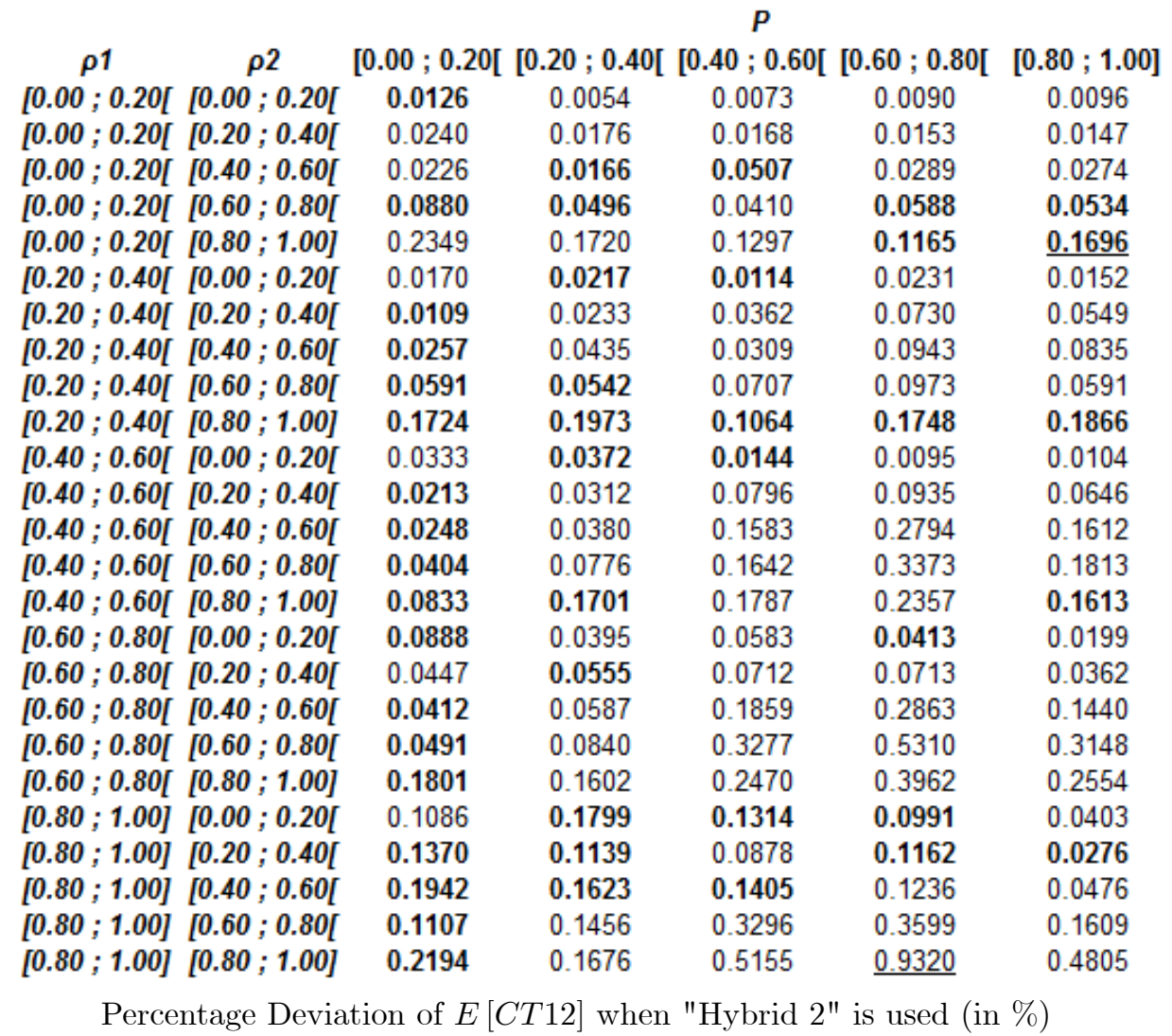




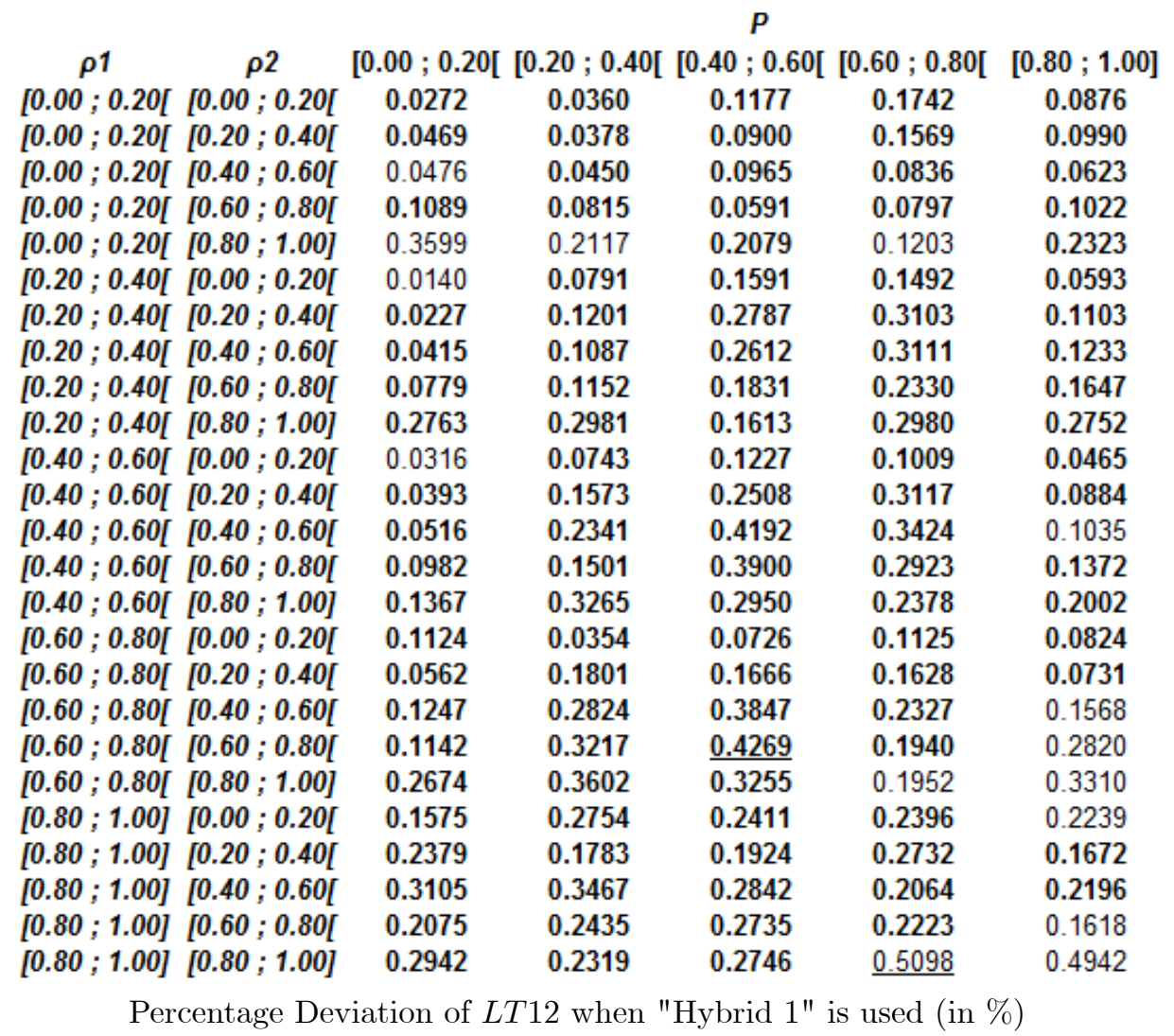




\section{P}

\begin{tabular}{ccccccc}
$\rho 1$ & $\rho 2$ & {$[0.00 ; 0.20[$} & {$[0.20 ; 0.40[$} & {$[0.40 ; 0.60[$} & {$[0.60 ; 0.80[$} & {$[0.80 ; 1.00]$} \\
{$[0.00 ; 0.20[[0.00 ; 0.20[$} & 0.0272 & 0.0360 & 0.1177 & 0.1743 & 0.0915 \\
{$[0.00 ; 0.20[[0.20 ; 0.40[$} & 0.0469 & 0.0378 & 0.0900 & 0.1569 & 0.1063 \\
{$[0.00 ; 0.20[[0.40 ; 0.60[$} & 0.0476 & 0.0450 & 0.0965 & 0.0836 & 0.0679 \\
{$[0.00 ; 0.20[[0.60 ; 0.80[$} & 0.1089 & 0.0815 & 0.0591 & 0.0797 & 0.1043 \\
{$[0.00 ; 0.20[[0.80 ; 1.00]$} & 0.3599 & 0.2117 & 0.2079 & 0.1203 & 0.2327 \\
{$[0.20 ; 0.40[[0.00 ; 0.20[$} & 0.0140 & 0.0791 & 0.1593 & 0.1506 & 0.0627 \\
{$[0.20 ; 0.40[[0.20 ; 0.40[$} & 0.0227 & 0.1202 & 0.2792 & 0.3166 & 0.1447 \\
{$[0.20 ; 0.40[[0.40 ; 0.60[$} & 0.0415 & 0.1087 & 0.2613 & 0.3159 & 0.1856 \\
{$[0.20 ; 0.40[[0.60 ; 0.80[$} & 0.0779 & 0.1152 & 0.1831 & 0.2343 & 0.2127 \\
{$[0.20 ; 0.40[[0.80 ; 1.00]$} & 0.2763 & 0.2981 & 0.1613 & 0.2981 & 0.3113 \\
{$[0.40 ; 0.60[[0.00 ; 0.20[$} & 0.0316 & 0.0745 & 0.1238 & 0.1035 & 0.0495 \\
{$[0.40 ; 0.60[[0.20 ; 0.40[$} & 0.0393 & 0.1585 & 0.2596 & 0.3421 & 0.0890 \\
{$[0.40 ; 0.60[[0.40 ; 0.60[$} & 0.0516 & 0.2356 & 0.4359 & 0.4211 & 0.1476 \\
{$[0.40 ; 0.60[[0.60 ; 0.80[$} & 0.0982 & 0.1507 & 0.4006 & 0.3686 & 0.2626 \\
{$[0.40 ; 0.60[[0.80 ; 1.00]$} & 0.1367 & 0.3265 & 0.2968 & 0.2666 & 0.3905 \\
{$[0.60 ; 0.80[[0.00 ; 0.20[$} & 0.1124 & 0.0351 & 0.0741 & 0.1132 & 0.0829 \\
{$[0.60 ; 0.80[[0.20 ; 0.40[$} & 0.0564 & 0.1863 & 0.1823 & 0.1806 & 0.0701 \\
{$[0.60 ; 0.80[[0.40 ; 0.60[$} & 0.1259 & 0.3057 & 0.4630 & 0.3757 & 0.1105 \\
{$[0.60 ; 0.80[[0.60 ; 0.80[$} & 0.1157 & 0.3603 & 0.5903 & 0.4472 & 0.1448 \\
{$[0.60 ; 0.80[[0.80 ; 1.00]$} & 0.2684 & 0.3742 & 0.4946 & 0.4437 & 0.3825 \\
{$[0.80 ; 1.00][0.00 ; 0.20[$} & 0.1575 & 0.2754 & 0.2412 & 0.2397 & 0.2240 \\
{$[0.80 ; 1.00][0.20 ; 0.40[$} & 0.2377 & 0.1797 & 0.1979 & 0.2778 & 0.1685 \\
{$[0.80 ; 1.00][0.40 ; 0.60[$} & 0.3147 & 0.3737 & 0.3172 & 0.2347 & 0.2263 \\
{$[0.80 ; 1.00][0.60 ; 0.80[$} & 0.2242 & 0.3437 & 0.4248 & 0.3503 & 0.1042 \\
{$[0.80 ; 1.00][0.80 ; 1.00]$} & 0.3124 & 0.4962 & $\underline{0.6100}$ & 0.2684 & $\underline{0.2413}$ \\
\hline
\end{tabular}

Percentage Deviation of LT12 when "Hybrid 2" is used (in \%) 